\title{
Eriodictyol ameliorated cognitive dysfunction in APP/PS1 mice through inhibited ferroptosis via vitamin D receptor mediated Nrf2 activation
}

\section{Lin Li}

The Third Affiliated Hospital of Chongqing Medical University

\section{Wenjun Li}

The Third affiliated hospital of Chongqing medical university

\section{Xiangru Zheng}

The Third Affiliated Hospital of Chongqing Medical University

\section{Qinglong Liu}

The third affiliated hospital of Chongqing medical university

\section{Qian Du}

The third affiliated hospital of Chongqing medical university

\section{Yujie Lai}

The Third Affiliated Hospital of Chongqing Medical University

\section{Songqing Liu ( $\sim$ liusq@hospital.cqmu.edu.cn )}

The Third Affiliated Hospital of Chongqing Medical University https://orcid.org/0000-0002-9269-3961

\section{Research article}

Keywords: Alzheimer's disease, Eriodictyol, Ferroptosis, VDR, Nrf2

Posted Date: November 11th, 2021

DOI: https://doi.org/10.21203/rs.3.rs-1046295/v1

License: (9) This work is licensed under a Creative Commons Attribution 4.0 International License. Read Full License 


\section{Abstract \\ Background}

Alzheimer's disease (AD) is the most common type of neurodegenerative disease in contemporary era, and it is still clinically incurable. Eriodictyol, a natural flavonoid compound mainly exists in citrus fruits and some Chinese herbal medicine, has been reported with its effect of anti-inflammatory, antioxidant, anti-cancer and neuroprotective effects. However, there are few studies on the anti-AD effect and molecular mechanism of eriodictyol.

\section{Methods}

APP/PS1 mice were treated with eriodictyol and the cognitive function of mice was assessed by behavioral tests. The level of amyloid- $\beta(A \beta)$ aggregation and hyper-phosphorylation of Tau in the brain of mice were detected by histological analysis and Western blotting. Meanwhile, HT-22 cells which induced by amyloid- $\beta$ peptide (1-42) $\left(A \beta_{1-42}\right)$ oligomer were treated with eriodictyol after which cell viability was determined and the production of $p$-Tau was tested by Western blotting. Then, the characteristics of ferroptosis, including iron aggregation, lipid peroxidation and the expression of glutathione peroxidase type 4(GPX4), were determined both in vivo and in vitro by Fe straining, Western blotting and APCR assay. Additionally, the expression level of Vitamin D receptor (VDR) and the activity of nuclear factor erythroid 2-related factor 2/heme oxygenase-1 (Nrf2/HO-1) signaling pathway were tested by Western blotting and qPCR assay. After that, the HT-22 cells with VDR knockout were used to explore the potential mechanisms and the relationship between VDR and Nrf2 was further assessed by coimmunoprecipitation assay and bioinformatics analysis.

\section{Results}

Eriodictyol obviously ameliorated cognitive deficits in APP/PS1 mice, suppressed A $\beta$ aggregation and the phosphorylated level Tau in the brain of APP/PS1 mice. Meanwhile, eriodictyol could inhibit Tau hyperphosphorylation and neurotoxicity in HT-22 cells induced by $A \beta_{1-42}$ oligomer. Furthermore, both in vivo and in vitro, eriodictyol showed the anti-ferroptosis effect and its mechanism may connected with the activation of Nrf2/HO-1 signaling pathway. Additionally, the further experiment explains that the activation of Nrf2/HO-1 signaling pathway with eriodictyol treatment mediated by VDR.

\section{Conclusions}

Eriodictyol alleviated memory impairment and AD-like pathological changes via activating Nrf2/HO-1 signaling pathway mediated by VDR, which provide a new possibility for the treatment of AD. 


\section{Background}

Alzheimer's disease (AD) is the most common progressive neurodegenerative disease. According to Word Alzheimer Report 2018, the number of AD patients will increase to 152 million by 2050 with the development of population aging[1]. Alzheimer's disease has become a severe social problem. However, there are no ideal therapeutic drugs and treatment for $A D$ so far.

Ferroptosis, first proposed by Dixon et al. in 2012, is a new form of non-apoptotic regulated cell death (RCD) which dependent on accumulation of intracellular iron and characterized by lipid peroxides[2]. Recently, an increasing number of findings suggested that ferroptosis plays a vital role in the development of AD. One research group found that ferroptosis which induced by loss of ferroportin-1 (Fpn, also be called as SLC40A1) play a critical role in the progression of AD, they explored that genetic deletion of Fpn in principal neurons of the neocortex and hippocampus led to AD-like hippocampal atrophy, memory deficits and the canonical morphological and molecular characteristics of ferroptosis[3]. Hambright WS et al. found that knockout of glutathione peroxidase type 4 (GPX4) in cerebral cortex and hippocampal neurons of mice induced degeneration of hippocampal neurons, resulting in obvious cognitive impairment. However, when mice were administered liproxstatin-1 (a ferroptosis inhibitor), the level of neurodegeneration was alleviated[4]. Additionally, a large cohort study found that ferroptosis brain iron burden at risk of accelerating the progression of $A D[5]$. These findings suggested that ferroptosis provides a promising research direction of developing anti-AD drugs.

Recently, many of natural products from plants or traditional medicines are showed great potentiality for $A D$ prevention and treatment, due to their multiple targets and low toxicity[6-8]. Especially, many flavonoids shown efficiently neuroprotective effects and become a hot research topic in preventing and treating Alzheimer's disease[9-11]. Eriodictyol (ERD, the chemical structure was shown in Fig. 1A), being the flavonoids compounds, exists in peel of citrus fruits and some Chinese herbal medicine widely[12]. Existing studies have shown that eriodictyol has a various of biological activities such as antiinflammatory, antioxidant and neuroprotective effects[13-16]. Xu Jing et al. had found that eriodictyol can attenuate neuronal cell death induced by $A \beta_{25-35}$ peptide[17]. Another study reported that eriodictyol improve LPS-induced cognitive impairments via inhibiting nuclear factor KB (NF-KB) in male C57BL/6J mice[18]. Nevertheless, at present, the molecular mechanism of eriodictyol's anti-AD effect has not been fully clarified.

In this study, we verified that eriodictyol has anti-AD effect using APPswe/PS1E9 transgenic mice and HT22 cell line. In addition, eriodictyol could inhibit ferroptosis in vitro and in vivo. Further, we explored the underlying mechanism and found that eriodictyol could activate the nuclear factor erythroid 2-related factor 2/ heme oxygenase-1 (Nrf2/HO-1) signaling pathway mediated by Vitamin D receptor (VDR). It illustrates that eriodictyol may be a potential drug for treatment of $A D$ and provides a regulating promising target of flavonoids compounds on Alzheimer's disease.

\section{Materials And Methods}




\section{Materials and Regents.}

Eriodictyol (purity > 95\%) was a gift from Syntech (SSPF) International, Inc (Montclair, CA). A $\beta_{1-42}$ [Amyloid $\beta$ Peptide (1-42), rat, J0426A] was purchased from Dalian Meilun Biotechnology, Co., Ltd. (Dalian, China). Iron Assay Kit, Congo Red Kit, Prussian blue, all-trans-retinoic acid (RA) and FITCphalloidin were obtained from Sigma-Aldrich (USA). Lipid Peroxidation MDA Assay Kit, Lipo8000, Propidium lodide Test Kit (including DAPI), ROS Assay Kit and Calcein-acetoxymethyl (Calcein-AM) were purchased from Beyotime Biotechnology, Co., Ltd. (Shanghai, China). Protein A/G Magnetic Beads for CoIP assay was purchased from Bimake (Houston, TX, United States). The Cell Counting Kit-8 (CCK-8), All-inOne cDNA Synthesis SuperMix Kit and 2×SYBR Green qPCR Master Mix (Low ROX) were obtained from Bimake (Houston, TX, United States). RNAsimple Total RNA Kit was purchased from TIANGEN Biotech Co., Ltd (Beijing, China). Anti-Phospho-Tau (S396) (A5952), anti-Ferritin (A5654), anti-GPX4 (A5569), and anti-Lamin B1 (A5106) antibodies also were obtained from Bimake (Houston, TX, United States). AntiSLC40A1 (26601-1-AP), anti-Beta Amyloid (25524-1-AP), and anti-Tau (66499-1-Ig) antibodies were bought from ProteinTech Group, Inc. (Chicago, IL, United States). Anti-Vitamin D Receptor (AF6159), antiNrf2 (AF0639), anti-Phospho-Nrf2 (Ser40) (DF7519), anti-HO-1 (AF5393), and anti-Transferrin Receptor (AF5343) antibodies were obtained from Affinity Biosciences (Zhenjiang, China). Goat anti-rabbit IgG H\&L (IRDye $800 \mathrm{CW}$ ) pre-adsorbed secondary antibody was obtained from Abcam (Cambridge, United Kingdom).

\section{Animals and Treatments.}

APPswe/PS1E9 transgenic mice (APP/PS1 mice, female, 9 months old), were obtained from Nanjing Junke Bioengineering Co., Ltd. (Nanjing, China) and wild-type C57 mice (female, 9 months old) were obtained from the Animal Experimental Center of Chongqing Medical University (Chongqing, China). All mice were bred in the Animal Experimental Center of Chongqing Medical University. All mice husbandry procedures performed were approved by the Chongqing Medical University Animal Welfare Committee. As shown in Fig. 1B, APP/PS1 transgenic mice were divided randomly into two groups (6 mice in per group): modle (saline, i.p., 3 times per week), ERD-treated group (50 mg/ $\mathrm{kg}$ ERD, i.p., 3 times per week); the wildtype C57 mice as positive control group (saline, i.p., 3 times per week). Mice body weights were measured three times a week. After three months of continuous administration, behavioral tests were conducted on mice. Then, all mice were humanely killed and the brains were harvested immediately for further analysis[6].

\section{Morris Water Maze Test.}

The Morris water maze test was carried out according to a previous protocol[19]. Performance was videorecorded and analyzed by image analyzing software (ANY-maze, Stoelting). In platform trials, the escape latency and swimming route were recorded. In probe trials, the swimming route, annulus crossings, the swimming distance and the time spent in each quadrant were recorded.

\section{Y-maze Test.}


The Y-maze test was carried out according to a previous protocol[20]. In the spontanenous alternation test, the number of total entries and spontanenous alternation of mice were recorded. The percentage of alternation was calculated using the following formula: [the number of spontanenous alternation/ (total number of arm entries -2)] $\times 100 \%[21]$. In the novel arm exploration test, the number of times the mice entered the novel arm, the distance traveled, the time spent in the novel arm of mice were recorded.

\section{Congo Red Staining.}

The $A \beta$ plaques in the brain of mice were stained by Congo red and photographed using a light microscope (Nikon, Janpan) according to the manufacturer's operating instructions[22].

\section{Fe Staining.}

Iron in the mice's brain was stained using Prussian blue following a previous protocol[23], and take photograph under a fluorescence microscopy (Nikon, Janpan) .

\section{Immunohistochemistry (IHE) analysis.}

IHE analysis of the brain tissues using anti-VDR and anti-A $\beta$ antibodies, and the steps refers to a previous study[24].

\section{Cell Culture and Preparation of $A \beta_{1-42}$.}

HT-22 cells which were from the Chinese Academy of Sciences (Shanghai, China) were cultured in Dulbecco's modified Eagle's medium (DMEM, (Biological Industries, Israel) supplemented with $10 \%$ FBS (Biological Industries, Israel) and 1\% penicillin/streptomycin (Beyotime Biotechnology, Shanghai). The cells were maintained in a sterile and humidified atmosphere at $37{ }^{\circ} \mathrm{C}$ with $5 \% \mathrm{CO}_{2}$. Eriodictyol was dissolved in dimethyl sulfoxide (DMSO) and the final concentration of DMSO in culture medium was $\leq$ $1 \%$. The preparation method of $A \beta_{1-42}$ oligomers is as follows: $A \beta_{1-42}$ was dissolved in DMSO and sonicated for 5 minutes in a cold-water bath, then immediately stored at $-80{ }^{\circ} \mathrm{C}$ as stock. The $A \beta_{1-42} / D M S O$ solution was diluted with serum-free DMEM to a final concentration of $100 \mu \mathrm{M}$ and stored at $37^{\circ} \mathrm{C}$ until use[25].

\section{Cell Viability Assay.}

The cell viability of HT-22 cells after treated with eriodictyol $(0-128 \mu \mathrm{mol} / \mathrm{L})$ and the role of eriodictyol in $A \beta_{1-42}$ oligomers-induced cellular damage were detected using CCK-8 assay according to the manufacturer's instructions, and the absorbance was measured at $450 \mathrm{~nm}$ by a SynergyH 1 microplate reader (BioTek).

Transmission Electron Microscopy (TEM) Imaging. 
HT-22 cells were collected after treatment for $48 \mathrm{~h}$ and fixed in $2.5 \%$ glutaraldehyde. Then each sample was made as slice according to a previous protocol[26]. The digital images were acquired using a JEM1400 PLUS transmission electron microscope (TEM).

\section{FITC-phalloidin Assay.}

FITC-phalloidin used to stain cytoskeleton of HT-22 cells. HT-22 cells was plated in 24-well plate and cultured with culture medium containing $10 \mu \mathrm{M}$ RA to induced neural axons formation for 3 days. Then $20 \mu \mathrm{M} \mathrm{A} \beta_{1-42}$ oligomers were added into culture medium after cells pre-treated with eriodictyol in different concentration for $2 \mathrm{~h}$, the vehicle control group only was added culture medium. $48 \mathrm{~h}$ later, the cells were fixed in $4 \%(\mathrm{wt} / \mathrm{vol})$ paraformaldehyde for $15 \mathrm{~min}$ and incubated with FITC-phalloidin solution and DAPI in turn at $37^{\circ} \mathrm{C}$. Finally, images were collected under a fluorescence microscope at 400x magnification and the length of axons was measured by ImageJ software[6].

\section{Propidium lodide (PI) Assay.}

$\mathrm{PI}$ assay was implemented according to the manufacturer's instructions. After that, cells were observed and taken pictures by a fluorescence microscope at 100x magnification[27].

\section{Calcein-acetoxymethyl (Calcein-AM) Assay.}

The level of iron in HT-22 cells were detected by Calcein-AM following the manufacturer's instructions[28]. Photographs were taken under fluorescence microscope [29].

\section{ROS Assay.}

HT-22 cells after treated were assessed intracellular ROS level using ROS Assay Kit according to the manufacturer's instructions. Observe and take photos under a fluorescence microscope at 100x magnification[30].

\section{Iron Assay.}

Iron concentration in the brain tissues and HT-22 cells were assessed according to the manufacturer's instructions of Iron Assay Kit.

\section{MDA Assay.}

The malondialdehyde (MDA) content in the brain of mice and HT-22 cells were assessed according to the manufacturer's instructions[22].

\section{Western Blot Analysis.}

Total protein of brain tissues or HT-22 cells was extracted. Nuclear and cytosolic proteins of HT-22 cells were extracted according to the manufacturer's instructions of the Nuclear and Cytoplasmic protein 
Extraction Kit[31]. According a previous protocol, the expression level of proteins was measured by Western blot assay[29, 32].

\section{Real-time Quantitative PCR (qPCR).}

According to the manufacturer's instructions of the Total RNA Extraction Kit, total RNA of HT-22 cells were extracted. Then, the total RNA was reverted in cDNA by using the All-in-One cDNA Synthesis SuperMix kit. Eventually, qPCR was performed with the $2 \times$ SYBR Green qPCR Master Mix to measure the expression of mRNA. The gene-specific primers were mentioned in Table 1. The expression values were normalized to the expression of $\beta$-actin and calculated with $2^{-\Delta \Delta C t}$ method[33].

Table 1

Primer sequences used for the qPCR analysis.

\begin{tabular}{|lll|}
\hline Gene & Forward primer, $\mathbf{5}^{\prime} \mathbf{3}^{\prime}$ & Reverse primer, $\mathbf{5}^{\prime} \mathbf{3}^{\prime}$ \\
\hline B-actin & GGCTGTATTCCCCTCCATCG & CCAGTTGGTAACAATGCCATGT \\
\hline TfRC & GCCTTGCTCGGCAAGTAGAT & TCCTCCGTTTCAGCCAGTTT \\
\hline FTH & AGAGCGGGCTGAATGCAATG & ATATTCTGCCATGCCAGCTTCAG \\
\hline Fpn & CCAAGGCAAGAGATCAAACCCA & CAGGATGATTCCGCAGAGGAT \\
VDR & ATGAGGAGGTGCAGCGTAAG & CATCGAGCAGGATGGCGATA \\
GPX4 & CCTTCCCCTGCAACCAGTTT & CCACGCAGCCGTTCTTATCA \\
\hline
\end{tabular}

\section{CRISPR/Cas9.}

CRISPR/Cas9 was used for VDR knockout (KO)[34]. The single-guide RNA (sgRNA), sequence: 5'AGTCTGGAAAGCGTCACTTG-3', was cloned into lenti-CRISPRV2 plasmid and co-transfected into HEK293T with psPAX2, pMD2.G and Lipo8000 in order to generate virus particles. Forty eight hours after being transfected, the virus particles in the cell culture fluid were filtered with filter membrane $(0.45 \mu \mathrm{m})$ and infected into HT22 cell line in the presence of Polybrene $(10 \mu \mathrm{g} / \mathrm{mL})$ at $37^{\circ} \mathrm{C}$. After $48 \mathrm{~h}$, Puromycin $(2 \mu \mathrm{g} / \mathrm{mL})$ was added to $\mathrm{KO}$ cells and NC cells to select stable infected cells. The efficiency of knockout was confirmed by Western blotting[35].

Coi

mmunoprecipitation (Co-IP) Assay.

Co-IP analysis was carried out according to the manufacturer's instructions of protein A/G magnetic beads (B23201, Bimake, China, Shanghai)[36, 37].

Bioinformatics Analysis. 
All microarray data were downloaded from gene expression omnibus (GEO) database (accession no. GSE48350). The raw data were downloaded as MINiML files. The extracted data were normalized and processed by log2 transformation. The microarray data were normalized using the preprocessCore package in R software (version 3.4.1). Differentially expressed genes were identified using DESeq2 with the adjusted $P$ value $<0.01$. Gene expression correlation was analyzed with Spearman test.

\section{Statistical Analysis.}

GraphPad Prism 8.0.1 software was adopted for statistical analyses. All data were analyzed by Student $t$ tests. $P<0.05$ was considered statistically significant. All data are the means $\pm \mathrm{SD}$, and the tests were repeated in at least three independent experiments.

\section{Results}

\section{Eriodictyol ameliorates cognitive deficits in APP/PS1 mice.}

First at all, we carried out Morris water maze experiment to test the spatial learning and memory function of mice. As shown in Fig. 1G, there was no difference in the mean swimming speed of all group mice, which means, eriodictyol treatment did not affect the basic motor ability of APP/PS1 mice. On the fifth day of platform trials, the escape lantency of APP/PS1 mice was longer compared with WT mice, while eriodictyol treatment significantly reduced the escape lantency of APP/PS1 mice (Fig. 1C). Next, the platform was removed to carry out a probe trails. The typical trajectories of each group mice are shown in Fig. $1 \mathrm{H}$. For APP/PS1 mice, the swimming distance and the time spent in the target quadrant were shorter and the number of platform crossings was fewer than WT mice, however, when APP/PS1 mice was intraperitoneally injected with eriodictyol, the proportion of distance and the time spent in the target quadrant was dramatically lengthened and the number of platform crossings was increased (Fig. 1D-F). As expected, the results of Morris water maze were suggested that APP/PS1 mice show significant spatial learning and memory deficits. However, eriodictyol treatment effectively improved these deficits in APP/PS1 mice.

Subsequently, to further investigate whether eriodictyol had a positive effect on memory function of APP/PS1 mice, Y-maze tests were carried out after completion of Morris water maze tests. Consistent with the results of the Morris water maze tests, the results of $Y$-maze tests showed that cognitive impairment in APP/PS1 mice was significantly ameliorated by eriodictyol treatment. To be specific, in spontaneous alternation tests, compared with WT mice, the percentage of spontaneous alternation and the number of total entries within 8 minutes were reduced in APP/PS1 mice, while eriodictyol memorably reversed it (Fig. 2A-B). Then in novel arm exploration tests, we also found that the APP/PS1 mice treated with eriodictyol showed a higher percentage of novel arm entries, more time spent in the novel arm and more number of total entries within 5 minutes compared with the mice in APP/PS1-control group (Fig. 2CE). The representative track of each group of mouse is demonstrated in Fig. 2F. These results of behavioral tests were indicated that eriodictyol can ameliorate cognitive deficits in aged APP/PS1 mice. 


\section{Eriodictyol alleviates A $\beta$ aggregation and Tau hyper-phosphorylation in APP/PS1 mice.}

To investigate whether eriodictyol had a beneficial effect on $A \beta$ aggregation and Tau hyperphosphorylation which are in APP/PS1 mice, we performed Congo red staining for amyloid plaques, IHE analysis for $A \beta$ protein and phosphorylated-Tau (p-Tau). The result of Congo red staining was shown in Fig. 3A, we observed that eriodictyol was markedly reduce $A \beta$ aggregation in the brain of APP/PS1 mice. Similarly, IHE assay revealed that there was a marked reduction of $A \beta$ protein in the brain of eriodictyoltreated APP/PS1 mice, and the level of p-Tau also reduced by eriodictyol treatment (Fig. 3B). Then, the results of Western blot assay agreed with above studies, the expression level of $A \beta$ and $p$-Tau in the cortex and hippocampus were reduced by eriodictyol compared with APP/PS1 control mice, despite there was no remarkable change of Tau (Fig. 3C-D). These fundings revealed that eriodictyol treatment alleviates $A \beta$ aggregation and Tau hyper-phosphorylation in APP/PS1 mice.

\section{Eriodictyol inhibits ferroptosis in brain of APP/PS1 mice.}

Iron accumulation in brain of mice was test using Prussian blue staining. As expected, more iron deposits which stained blue spots by Prussian blue were observed in brain of APP/PS1 mice, while treatment of eriodictyol could effectively reduce the level of iron in APP/PS1 mice (Fig. 4A). Meanwhile, the level of ferrous iron and total iron in cortex and hippocampus of mice were dectected using Iron Assay Kit. The result was shown in Fig. 4B, which suggested that compared with APP/PS1 mice, eriodictyol treatment significantly decreased the level of ferrous iron and total iron in cortex and hippocampus of APP/PS1 mice. Western blot analysis demonstrated that the expression of transferrin receptor (TfRC) and ferritin heavy chain (FTH) were reduced upon eriodictyol treatment in cortex and hippocampus of APP/PS1 mice compared with APP/PS1 control mice. While, Fpn (the only iron export protein) was up-regulated in cortex and hippocampus by eriodictyol treated (Fig. 4C). These data imply that eriodictyol treatment could maintain iron balance in cells through reducing iron intake and increasing iron output.

MDA is a specific marker of lipid peroxidation[6]. It can be found from Fig. 4D that MDA content in cortex and hippocampus of APP/PS1 mice was significantly higher than that of WT mice, while treatment with eriodictyol could reverse this tendency.

Finally, we investigated the expression of GPX4 in brain. Western blot analysis confirmed that the expression level of GPX4 was significantly increased upon eriodictyol treatment both in cortex and hippocampus of APP/PS1 mice (Fig. 4E).

Together, these results confirmed that eriodictyol effectively inhibited ferroptosis in brain cells of $A D$ model mice.

Eriodictyol attenuated the cytotoxicity and Tau hyper-phosphorylation induced by $A \beta_{1-42}$ oligomer in HT22 cells. 
HT-22 cell line was used to carry out relevant experiments in vitro. First at all, we determined that the dosage of $A \beta_{1-42}$ oligomer was $20 \mu \mathrm{M}$ to construct cell model of $A D$ according to relevant literatures[6, 21]. As shown in Fig. 5A, eriodictyol had no cytotoxicity to HT-22 cells, when the concentration was less than $32 \mu \mathrm{M}$.

In order to explore whether eriodictyol relieves the cytotoxicity of $A \beta_{1-42}$ oligomer in HT-22 cells, we carried out a series of study. The result showed that the cell viability of HT-22 cell was markedly decreased upon $A \beta_{1-42}$ oligomer induced, while eriodictyol treatment obviously improved the cell viability in a dose-dependent manner (Fig. 5B). Meanwhile, under the microscope, the corresponding changes were observed in the number of cells surviving (Fig. 5C). Then, FITC-phalloidin was applied to stain the cytoskeleton of HT-22 cells. In comparison with normal HT-22 cells, the axon length of HT-22 cells treated with $A \beta_{1-42}$ oligomer were visibly shortened by about $70 \%$, while supplementation of eriodictyol with different dose markedly reversed the effect of $A \beta_{1-42}$ oligomer (Fig. 5D-E). Additionally, Propidium lodide assay verify the antagonistic effect of eriodictyol on the cytotoxicity of $A \beta_{1-42}$ oligomer. As demonstrated in Fig. 5F, $20 \mu \mathrm{M} \mathrm{A} \beta_{1-42}$ oligomer induced HT-22 cells death, while after treated with eriodictyol in different concentration, the number of dead cells became obviously lower. Together, these results demonstrated that eriodictyol treatment relieves the cytotoxicity of $A \beta_{1-42}$ oligomer in HT-22 cells and dramatically maintains the morphology of neuronal cell. In addition, Western blot assay used to the expression level of $\mathrm{p}$-Tau and Tau. From the results, we found that the expression level of $\mathrm{p}$-Tau in HT-22 cells which had been exposed to $A \beta_{1-42}$ oligomer was increased compared with control group. Similar to the results in vivo, eriodictyol enormously inhibited hyper-phosphorylation of Tau protein induced by $A \beta_{1-42}$ oligomer, and a certain degree of dose-dependent (Fig. 5G).

\section{Eriodictyol inhibits ferroptosis induced by $A \beta_{1-42}$ oligomer in $\mathrm{HT}-22$ cells.}

The level of ferrous iron and total iron in HT-22 cell were tested using Iron Assay Kit, as can been seen from Fig. 6A, $A \beta_{1-42}$ oligomer treatment was associated with an about 2-fold increase in ferrous iron content and an about 1.5 -fold increase in total iron content. Conversely, eriodictyol notably reduced the increased iron aggregation induced by $A \beta_{1-42}$ oligomer.

Then, Calcein AM was used to stain cell to obverse the iron content. Cells is stained by Calcein AM and emits strong green fluorescence, while metal iron causes quench fluorescence. As shown in Fig. 6B, compared with control group, the green fluorescence was dimmer in HT-22 cells treated with $A \beta_{1-42}$ oligomer, while eriodictyol could enhance the fluorescence intensity. Western blot result showed that the expression level of proteins related to iron metabolism changed as expected (Fig. 6C). Compared with $A \beta_{1-42}$ oligomer-treated HT-22 cells, the expression level of TfRC and FTH were decreased in HT-22 cells treated with eriodictyol in a dose-dependent manner. On the contrary, the expression level of Fpn in HT-22 cells was up-regulated by eriodictyol, especially when the dose of eriodictyol was $8 \mu \mathrm{M}$. Meanwhile, qPCR assay results kept up with Western blot assay (Fig. 6D). 
Next, immunofluorometric assay was used to detect the intracellular ROS content in HT-22 cells. Compared with control group, the fluorescence intensity was higher in cells treated with $A \beta_{1-42}$ oligomer, while that was lower in cells under co-processing of $A \beta_{1-42}$ oligomer and eriodictyol (Fig. 6E). Expectedly, the MDA content in the cells was suppressed with treatment of eriodictyol (Fig. 6F).

What's more, the expression of GPX4, a specific ferroptosis-related gene, was measured by Western blot analysis and qPCR assay. From these results, the protein and mRNA expression of GPX4 in HT-22 cells were down-regulated by $A \beta_{1-42}$ oligomer, while eriodictyol treatment could up-regulate the protein and mRNA expression of GPX4 (Fig. 6G-H).

Additionally, the morphological features of $A \beta_{1-42}$ oligomer-treated $\mathrm{HT}-22$ cells were observed under electron microscope, including mitochondria appeared rupture and fragmentation with the increasing of membrane density, which consistent with characteristic of ferroptosis reported in Dixon's study[2]. Conversely, eriodictyol reversed the injury of mitochondrions induced by $A \beta_{1-42}$ oligomer (Fig. 6I).

\section{Eriodictyol up-regulates the expression of VDR and activates Nrf2/HO-1 signaling pathway.}

Some researchers discovered that nuclear factor erythroid 2-related factor 2 (Nrf2) is the key regulator of ferroptosis[38],[39]. Moreover, recent study reported that VDR was involved in ferroptosis, and VDR activation could inhibit ferroptosis[40]. In our study, IHE analysis showed the expression level of VDR was reduced in APP/PS1 mice compared with WT mice. In contrast, the expression of VDR in APP/PS1 mice was improved by eriodictyol treatment (Fig. 7A). The results of Western blot were consistent with IHE analysis (Fig. 7B). Then, the expression level of VDR in HT-22 cells was detected by Western blot and qPCR assay, and the result was consistent with vivo (Fig. 7. C-D).

We further tested the changes of Nrf2/HO-1 signaling pathway. As expected, the expression of phosphorylation of Nrf2 and HO-1 were up-regulated in cortex and hippocampus of APP/PS1 mice upon eriodictyol treatment compared with APP/PS1-control group, while the expression of total Nrf2 was no clear difference. Consistent with the vivo results, $A \beta_{1-42}$ oligomer treatment not only weakened the expression of phosphorylated-Nrf2 (p-Nrf2) and HO-1, but also inhibited Nrf2 enter into nucleus. However, when the HT-22 cells were treated with $A \beta_{1-42}$ oligomer and eriodictyol together, the expression level of $p$ Nrf2 and HO-1 were up-regulated, Nrf2 entering the nucleus also was increased (Fig. 7F-G). These data were suggested that eriodictyol promoted the expression of VDR and activated $\mathrm{Nrf} / \mathrm{HO}-1$ signaling pathway.

\section{VDR is the key mediator in the regulation of $\mathrm{Nrf} / \mathrm{HO}-1$ signaling pathway by eriodictyol.}

All the above results indicated that VDR and Nrf2/HO-1 signaling pathway participate in the realization of anti-ferroptosis of eriodictyol, but it is incompletely understood that the relationship of VDR and Nrf2/HO1 signaling pathway. To further understand whether VDR play an indispensable role in the activation of Nrf2/HO-1 signaling pathway by eriodictyol, we used CRISPR/CAS9 technology to knockout VDR in HT-22 cells (Fig. 8A). Then, the sgVDR HT-22 cell was used for CCK-8 assay with normal group. Eriodictyol 
relieved the reduction of cell viability induced by $A \beta_{1-42}$ oligomer, while VDR knockout reversed the protect effect of eriodictyol (Fig. 8B). In addition, we tested the expression of proteins related to $A D$ and ferroptosis. The results were showed in Fig. 8C, the phosphorylation of Tau, was partly restrained in HT-22 cells by eriodictyol treatment compared with $A \beta_{1-42}$ oligomer-treated group, but when the VDR was knocked out, the effect of eriodictyol was attenuated. Meanwhile, the facilitation effect of eriodictyol on GPX4 expression was reversed by VDR knockout. Such results suggested that VDR knockout markedly reversed the positive effects of eriodictyol on the HT-22 cell.

Subsequently, we found that knockout VDR blocked the promoting effect of eriodictyol on $\mathrm{Nrf} 2 / \mathrm{HO}-1$ signaling pathway from the results of Western blot assay (Fig. 8C-D). Specifically, the expression p-Nrf2 and HO-1 were reduced and nuclear translocation of Nrf2 was weakened in HT-22 cells with sgVDR. In addition, to further measured the interaction between VDR and Nrf2, Co-IP assay was carried out. The results confirmed that VDR could interact with Nrf2, and eriodictyol could improve the protein-protein interaction (Fig. 8E).

Finally, to explore the correlation between Nrf2 and VDR in AD patients, bioinformatics analysis was carried out. Gene difference analysis found that there were significant differences in the expression of GPX4 in cortex and hippocampus between normal elderly and AD patients, as shown in Fig. 8F. Whereas, the expression levels of VDR didn't show an obvious difference between the two groups. Next, correlation analysis showed that there was a positive tendency correlation between the expression of Nrf2 and VDR (Fig. 8G). These results indicated that VDR is a necessary factor for activating Nrf2/HO-1 signaling pathway and eriodictyol reduces memory impairment Alzheimer's disease by inhibiting ferroptosis, its mechanism is connected with up-regulation of Nrf2/HO-1 pathway which mediated by VDR.

\section{Discussion}

In recent years, the number of $A D$ patients has increased sharply in the world, and Alzheimer's disease has become an important factor affecting the quality of life for the aged[1]. Unfortunately, due to the complex pathogenesis and diverse symptoms of $A D$, there are no effective drugs to treat or prevent $A D$ until now. Eriodictyol, a natural flavonoid compound, has showed multiple biological activities, such as anti-inflammatory, antioxidant, anti-radical and neuroprotective effect $[13,14,16,17]$. However, the mechanism of eriodictyol in AD remains ambiguous. In this study, we found that eriodictyol could alleviate cognitive impairment in APP/PS1 mice and reduced pathological changes of AD both in vivo and vitro. For mechanism, our study implied that the anti-AD effect of eriodictyol was connected with inhibiting ferroptosis of neural cells, which via activating Nrf2/HO-1 signaling pathway mediated by VDR.

Ferroptosis is a novel form of programmed cell death, which have three main characteristics of ferroptosis, including abnormal accumulation of iron, lipid peroxidation, and decrease of GPX4[2] [41]. Firstly, accumulation of intracellular iron is connected with the transport of extracellular iron and the release of intracellular iron. To be specific, the expression of transferrin (Tf), TfRC and ferritin (indicated by FTH) were increased, while the expression of Fpn, which could transport iron out of cells, was 
decreased[2]. Secondly, lipid peroxidation showed the increase of MDA and ROS production[41, 42]. Thirdly, GPX4 is the key upstream regulator of ferroptosis[43], and studies found that deletion of GPX4 happens in brain which is suffered ferroptosis, which will damage biological macromolecules such as lipids and proteins $[44,45]$. Up to this point, more and more evidences support that ferroptosis participates in $A D[46,47]$. There are many studies found that iron accumulates is preferentially observed in the cortex and hippocampus of AD patients using magnetic resonance imaging (MRI)[48-54], and iron modulates the cleavage of APP and the hyper-phosphorylation of Tau[55]. When iron is overload, the levels of furin protein are reduced, which up-regulates $\beta$-secretase activity and then promotes the amyloidogenesis processing of APP, it causes APP depletion and A $\beta$ deposition[56-58]. Meanwhile, APP can also stabilize Fpn on the cell membrane to facilitate the efflux of iron from neurons and APP depletion can lead to iron accumulation in cultured neurons and in mouse models[59-61]. Additionally, GPX4 activity is of paramount importance in promoting or enabling neuronal survival[62]. This importance is graphically illustrated by an experiment which indicates ablation of GPX4 leads to the rapid degeneration of motor neurons[63]. Hambright et al. also found that GPX4 knockout markedly induces degeneration of hippocampal neurons and cognitive impairment in mice[4]. What's more, the Gene difference analysis in our study showed that the expression level of GPX4 in cortex and hippocampus of AD patients was significantly lower than normal elderly. Therefore, it points out that ferroptosis as a potential target for treatment or prevention of AD. In our study, we also found that the morphologic and biochemical hallmarks of ferroptosis appeared in the brain of APP/PS1 mice and A $\beta_{1-42}$ oligomer-treated HT-22 cells while eriodictyol could alleviate these changes (Fig. 4 and Fig. 6). To be specific, eriodictyol significantly reduced the accumulation of intracellular iron by down-regulated TfRC and FTH, up-regulated Fpn, and decreased the the content of MDA and ROS, as well as activated the expression of GPX4. These researches verified that ferroptosis was closely related to the pathologies and dependent of $A D$ and suggested that eriodictyol plays an anti-AD role by suppressing ferroptosis.

There are growing evidences shown that $\mathrm{Nrf} 2$ and its related signaling pathways play a necessary regulatory role in the process of ferroptosis[64,65]. On the one hand, Nrf2 can maintain cellular iron homeostasis by regulating the expression and activity of ferritin, Tf, TfRC, divalent metal-iron transporter1 (DMT1), nuclear receptor coactivator 4 (NCOA4), Fpn, and other related proteins and regulatory factors in the process of iron metabolism[64,66,67]. On the other hand, Nrf2 not only regulates the expression and activity of antioxidant enzymes in antioxidant system, such as superoxide dismutase (SOD), HO$1[39,66]$, but also improve the expression of GPX4 by maintain the level of glutathione in cells[38, 68]. Recently, researchers found that Nrf2 activation could endow on cells with anti-ferroptosis ability. For instance, Yanan Zhao et al. found that knockdown Nrf2 sensitized cell to ferroptosis by down-regulating the expression of Fpn and HO-1 and then reversed the protection of autophagy inhibition in alcoholinduced HepG2 cell[69]. The importance of Nrf2 in ferroptosis was verified again in the research carried out by Shuangwen Li's team[70]. Therefore, activating Nrf2 would seem worthy of consideration to inhibit ferroptosis in cells. Additionally, a number of researches proclaimed that the anti-AD effect of eriodictyol was related with activation of Nrf2/HO-1 signaling pathway. For examples, Lv P et al. found that eriodictyol could protect the RGC-5 cells from high glucose-induced oxidative stress, inflammation, and 
cell apoptosis by activating the Nrf2/HO-1 signaling pathway[71]. Aother study suggested that eriodictyol could protect neurons from the toxic damage of $A \beta_{25-35}$ by up-regulating Nrf2/ARE signaling pathway[72]. In our research, we also observed that eriodictyol inhibited pathology features of AD by activating Nrf2/HO-1 signaling pathway, including promoting the expression of p-Nrf2 nuclear translocation of Nrf2, thereby up-regulating the expression of HO-1. Furthermore, a previous study demonstrated that VDR (a karyophilic protein, which mediates $1,25(\mathrm{OH})_{2} \mathrm{D}_{3}$ to realize the biological functions[73]) also was associated with ferroptosis, it proposed that VDR activation can reverse ferroptosis-related changes in cisplatin induced AKI by trans-regulation of GPX4[40]. The correlation between VDR and ferroptosis also was verified in our study. The results indicated that eriodictyol induced a significant increasing of VDR expression both in vivo and in vitro with a dose-dependent manner (Fig. 7). Then, Gene difference analysis of the expression level of VDR in AD patients and the normal aged didn't apparent difference, maybe it's because the number of samples is too small (Fig. 8F). In 2018, a team reported that the promoter sequence of Nrf2 gene contains the VDR binding sites, and proved that Nrf2 transcriptional activation could be promoted by VDR[74]. Automatically, a question was posed: does eriodictyol activate Nrf2/HO-1 signaling pathway mediated by VDR? Our follow-up studies got the answer to this question. In our researches, we found that VDR knockout in HT-22 cells reversed the protective effect and anti-ferroptosis of eriodictyol. Then, the results of Co-IP assay also proved that VDR interacts with Nrf2. Besides, bioinformatic analysis verified that VDR has a positive correlation trend with Nrf2. These founding suggested that VDR play an indispensable role in activation of $\mathrm{Nrf} 2 / \mathrm{HO}-1$ pathway by eriodictyol.

According our research, a hypothesis can be put forward (Fig. 9): eriodictyol alleviated cognitive injury by preventing neuron cell from ferroptosis, its mechanism may be connected with the activation of Nrf2/HO1 mediated by VDR. However, there are shortages in this research. Firstly, we cannot explore the the antiAD effect of oral administration of eriodictyol in APP/PS1 mice. Secondly, it is not verified that eriodictyol how up-regulate VDR in this study. These researches will carry out in the follow-up study.

\section{Abbreviations}

AD, Alzheimer disease; ERD, eriodictyol; APP/PS1 mice, APPswe/PS1E9 transgenic mice; WT mice, wildtype $\mathrm{C} 57$ mice; i.p., intraperitoneal injection; $H T-22$ cell, mouse hippocampal neuron cell line; $A \beta$, amyloid$\beta$; Nrf2, nuclear factor erythroid 2-related factor 2; HMOX1 or HO-1, heme oxygenase-1;VDR, Vitamin D receptor; $\mathrm{p}$-Tau, phosphorylated-Tau; TfRC, transferrin receptor; FTH, ferritin heavy chain; p-Nrf2, phosphorylated-Nrf2; Fpn, ferroportin-1; GPX4; glutathione peroxidase type 4; ROS, reactive oxygen species; MDA, malondialdehyd; DHE, dihydroethidium; DCFH-DA, dihydrodichlorofluorescein diacetate.

\section{Declarations}

\section{Ethics approval and consent to participate}


All animal experiments were conducted with the approval of Chongqing Medical University Animal Welfare Committee.

\section{Consent for publication}

Not applicable.

\section{Availability of data and materials}

The datasets used and/or analysed during the current study are available from the corresponding author on reasonable request.

\section{Competing interests}

All financial and non-financial competing interests must be declared in this section.

\section{Founding}

This work was supported by Application and Development Project from Chongqing Science and Technology Commission (No. cstc2020jscx-msxmX0123), Chongqing Basic Research and Frontier Exploration Project (No. cstc2018jcyjAX0170) and National Natural Science Foundation of China (No. 81801071).

\section{Authors' contributions}

SQL, WJL, and YJL conceived of and designed the research; LL and WJL performed the experiments; QLL analyzed the data; XRZ provided guidance of CRISPR/Cas9; QD prepared the figures; LL drafted the manuscript. All authors read and approved the final manuscript.

\section{Acknowledgements}

Not applicable.

\section{Author details}

${ }^{1}$ Department of Pharmacy, The Third Affiliated Hospital of Chongqing Medical University, Chongqing, 401120, PR China

2 Department of Hepatobiliary and Pancreatic Surgery, The Third Affiliated Hospital of Chongqing Medical University, Chongqing, 401120, PR China

${ }^{3}$ Department of Neurology, The Third Affiliated Hospital of Chongqing Medical University, Chongqing, 401120, PR China

\section{References}


1. Patterson C: World Alzheimer Report 2018: The state of the art of dementia research: New frontiers. In. London: Alzheimer's Disease International; 2018.

2. Dixon Scott J, Lemberg Kathryn M, Lamprecht Michael R, Skouta R, Zaitsev Eleina M, Gleason Caroline E, Patel Darpan N, Bauer Andras J, Cantley Alexandra M, Yang Wan S et al: Ferroptosis: An IronDependent Form of Nonapoptotic Cell Death. Cell 2012, 149(5):1060-1072.

3. Bao WD, Pang P, Zhou XT, Hu F, Xiong W, Chen K, Wang J, Wang F, Xie D, Hu YZ et al: Loss of ferroportin induces memory impairment by promoting ferroptosis in Alzheimer's disease. Cell Death Differ 2021, 28(5):1548-1562.

4. Hambright WS, Fonseca RS, Chen L, Na R, Ran Q: Ablation of ferroptosis regulator glutathione peroxidase 4 in forebrain neurons promotes cognitive impairment and neurodegeneration. Redox Biol 2017, 12:8-17.

5. Ayton S, Portbury S, Kalinowski P, Agarwal P, Diouf I, Schneider JA, Morris MC, Bush Al: Regional brain iron associated with deterioration in Alzheimer's disease: A large cohort study and theoretical significance. Alzheimer's \& dementia : the journal of the Alzheimer's Association 2021, 17(7):1244-1256.

6. Jiao S-S, Yao X-Q, Liu Y-H, Wang Q-H, Zeng F, Lu J-J, Liu J, Zhu C, Shen L-L, Liu C-H et al: Edaravone alleviates Alzheimer's disease-type pathologies and cognitive deficits. Proceedings of the National Academy of Sciences 2015, 112(16):5225-5230.

7. Wang JH, Lei X, Cheng XR, Zhang XR, Liu G, Cheng JP, Xu YR, Zeng J, Zhou WX, Zhang YX: LWAFC, a new formula derived from Liuwei Dihuang decoction, ameliorates behavioral and pathological deterioration via modulating the neuroendocrine-immune system in PrP-hAbetaPPswe/PS1(DeltaE9) transgenic mice. Alzheimers Res Ther 2016, 8(1):57.

8. $\quad$ Pan RY MJ, Kong XX, Wang XF, Li SS, Qi XL, Yan YH, Cheng J, Liu Q, Jin W, Tan CH, Yuan Z.: Sodium rutin ameliorates Alzheimer's disease-like pathology by enhancing microglial amyloid- $\beta$ clearance. Sci Adv 2019, 5(2):eaau6328.

9. Yang W, Zhang J, Shi L, Ji S, Yang X, Zhai W, Zong H, Qian Y: Protective effects of tanshinone IIA on SH-SY5Y cells against oAbeta1-42-induced apoptosis due to prevention of endoplasmic reticulum stress. Int J Biochem Cell Biol 2019, 107:82-91.

10. Uddin MS, Kabir MT, Tewari D, Mathew B, Aleya L: Emerging signal regulating potential of small molecule biflavonoids to combat neuropathological insults of Alzheimer's disease. Sci Total Environ 2020, 700:134836.

11. Li Zhu ZX-B, Gu Juan-Hua, Zeng Yue-Qin, Li Jin-Tao.: Breviscapine exerts neuroprotective efects through multiple mechanisms in APP/PS1 transgenic mice. Molecular and Cellular Biochemistry 2020, 468. 

eriodictyol to Jun-N terminal kinase and its anti-inflammatory signaling pathway. BMB Rep 2013, 46(12):594-599.

13. Wang Z, Lan Y, Chen M, Wen C, Hu Y, Liu Z, Ye L: Eriodictyol, Not Its Glucuronide Metabolites, Attenuates Acetaminophen-Induced Hepatotoxicity. Mol Pharm 2017, 14(9):2937-2951.

14. Wang X, Deng R, Dong J, Huang L, Li J, Zhang B: Eriodictyol ameliorates lipopolysaccharideinduced acute lung injury by suppressing the inflammatory COX-2/NLRP3/NF-kappaB pathway in mice. $J$ Biochem Mol Toxicol 2020, 34(3):e22434.

15. Bai J, Wang Y, Zhu X, Shi J: Eriodictyol inhibits high glucose-induced extracellular matrix accumulation, oxidative stress, and inflammation in human glomerular mesangial cells. Phytother Res 2019, 33(10):2775-2782.

16. He P, Yan S, Wen X, Zhang S, Liu Z, Liu X, Xiao C: Eriodictyol alleviates lipopolysaccharidetriggered oxidative stress and synaptic dysfunctions in BV-2 microglial cells and mouse brain. $J$ Cell Biochem 2019, 120(9):14756-14770.

17. Jing X SH, Zhu X, Wei X, Ren M, Han M, Ren D, Lou H. : Eriodictyol Attenuates $\beta$-Amyloid 25-35 Peptide-Induced Oxidative Cell Death in Primary Cultured Neurons by Activation of Nrf2. Neurochem Res $2015 \mathrm{Jul}, 40(7): 1463-1471$.

18. He P, Yan S, Zheng J, Gao Y, Zhang S, Liu Z, Liu X, Xiao C: Eriodictyol Attenuates LPS-Induced Neuroinflammation, Amyloidogenesis, and Cognitive Impairments via the Inhibition of NF-kappaB in Male C57BL/6J Mice and BV2 Microglial Cells. J Agric Food Chem 2018, 66(39):10205-10214.

19. Lai YJ, Zhu BL, Sun F, Luo D, Ma YL, Luo B, Tang J, Xiong MJ, Liu L, Long Y et al: Estrogen receptor alpha promotes Cav1.2 ubiquitination and degradation in neuronal cells and in APP/PS1 mice. Aging Cel/ 2019, 18(4):e12961.

20. L Lin K, Sze SC, Liu B, Zhang Z, Zhang Z, Zhu P, Wang Y, Deng Q, Yung KK, Zhang S: 20(S)protopanaxadiol and oleanolic acid ameliorate cognitive deficits in APP/PS1 transgenic mice by enhancing hippocampal neurogenesis. J Ginseng Res 2021, 45(2):325-333.

21. Ding B, Lin C, Liu Q, He Y, Ruganzu JB, Jin H, Peng X, Ji S, Ma Y, Yang W: Tanshinone IIA attenuates neuroinflammation via inhibiting RAGE/NF-kappaB signaling pathway in vivo and in vitro. $J$ Neuroinflammation 2020, 17(1):302.

22. Rahman SO, Panda BP, Parvez S, Kaundal M, Hussain S, Akhtar M, Najmi AK: Neuroprotective role of astaxanthin in hippocampal insulin resistance induced by Abeta peptides in animal model of Alzheimer's disease. Biomed Pharmacother 2019, 110:47-58. 
23. Jahanshahi M, Khalili M, Margedari A: Naringin Chelates Excessive Iron and Prevents the Formation of Amyloid-Beta Plaques in the Hippocampus of Iron-Overloaded Mice. Front Pharmaco/2021, 12:651156.

24. Streit WJ, Braak H, Del Tredici K, Leyh J, Lier J, Khoshbouei H, Eisenloffel C, Muller W, Bechmann I: Microglial activation occurs late during preclinical Alzheimer's disease. Glia 2018, 66(12):2550-2562.

25. Sarlak Z, Moazzami M, Attarzadeh Hosseini M, Gharakhanlou R: The effects of aerobic training before and after the induction of Alzheimer's disease on ABCA1 and APOE mRNA expression and the level of soluble Abeta1-42 in the hippocampus of male Wistar rats. Iran J Basic Med Sci 2019, 22(4):399-406.

26. Hsieh $\mathrm{CH}$, Hsieh HC, Shih FS, Wang PW, Yang LX, Shieh DB, Wang YC: An innovative NRF2 nanomodulator induces lung cancer ferroptosis and elicits an immunostimulatory tumor microenvironment. Theranostics 2021, 11(14):7072-7091.

27. Huynh DTN, Jin Y, Myung C-S, Heo K-S: Ginsenoside Rh1 Induces MCF-7 Cell Apoptosis and Autophagic Cell Death through ROS-Mediated Akt Signaling. Cancers 2021, 13(8).

28. Li D, Liu B, Fan Y, Liu M, Han B, Meng Y, Xu X, Song Z, Liu X, Hao Q et al: Nuciferine protects against folic acid-induced acute kidney injury by inhibiting ferroptosis. British Journal of Pharmacology 2021, 178(5):1182-1199.

29. Prah J, Winters A, Chaudhari K, Hersh J, Liu R, Yang SH: Cholesterol sulfate alters astrocyte metabolism and provides protection against oxidative stress. Brain Res 2019, 1723:146378.

30. Ge MH, Tian H, Mao L, Li DY, Lin JQ, Hu HS, Huang SC, Zhang CJ, Mei XF: Zinc attenuates ferroptosis and promotes functional recovery in contusion spinal cord injury by activating Nrf2/GPX4 defense pathway. CNS Neurosci Ther 2021.

31. Yoo JM, Lee BD, Sok DE, Ma JY, Kim MR: Neuroprotective action of N-acetyl serotonin in oxidative stress-induced apoptosis through the activation of both TrkB/CREB/BDNF pathway and Akt/Nrf2/Antioxidant enzyme in neuronal cells. Redox Biol 2017, 11:592-599.

32. Li W, Du Q, Li X, Zheng X, Lv F, Xi X, Huang G, Yang J, Liu S: Eriodictyol Inhibits Proliferation, Metastasis and Induces Apoptosis of Glioma Cells via PI3K/Akt/NF-kB Signaling Pathway. Frontiers in Pharmacology 2020, 11.

33. Orlandella FM, Smaldone G, Salvatore G, Vitagliano L, Cianflone A, Parasole R, Beneduce G, Menna G, Salvatore M, Mirabelli P: The IncRNA TEX41 is upregulated in pediatric B-Cells Acute Lymphoblastic Leukemia and it is necessary for leukemic cell growth. Biomark Res 2021, 9(1):54.

34. Jin HR, Du CH, Wang CZ, Yuan CS, Du W: Ginseng metabolite Protopanaxadiol induces Sestrin2 expression and AMPK activation through GCN2 and PERK. Cell Death Dis 2019, 10(4):311. 
35. Qu X, Yan X, Kong C, Zhu Y, Li H, Pan D, Zhang X, Liu Y, Yin F, Qin H: c-Myb promotes growth and metastasis of colorectal cancer through c-fos-induced epithelial-mesenchymal transition. Cancer Science 2019, 110(10):3183-3196.

36. Yu S, Zhang Y, Li Q, Zhang Z, Zhao G, Xu J: CLDN6 promotes tumor progression through the YAP1-snail1 axis in gastric cancer. Cell Death Dis 2019, 10(12):949.

37. Wang Z, Sun R, Wang G, Chen Z, Li Y, Zhao Y, Liu D, Zhao H, Zhang F, Yao J et al: SIRT3-mediated deacetylation of PRDX3 alleviates mitochondrial oxidative damage and apoptosis induced by intestinal ischemia/reperfusion injury. Redox Bio/ 2020, 28:101343.

38. Chen D, Tavana O, Chu B, Erber L, Chen Y, Baer R, Gu W: NRF2 Is a Major Target of ARF in p53Independent Tumor Suppression. Mol Cell 2017, 68(1):224-232 e224.

39. Liu Q, Wang K: The induction of ferroptosis by impairing STAT3/Nrf2/GPx4 signaling enhances the sensitivity of osteosarcoma cells to cisplatin. Cell Biology International 2019, 43(11):1245-1256.

40. Hu Z, Zhang H, Yi B, Yang S, Liu J, Hu J, Wang J, Cao K, Zhang W: VDR activation attenuate cisplatin induced AKI by inhibiting ferroptosis. Cell Death Dis 2020, 11(1):73.

41. Lei P, Bai T, Sun Y: Mechanisms of Ferroptosis and Relations With Regulated Cell Death: A Review. Front Physio/ 2019, 10:139.

42. Xie Y, Hou W, Song X, Yu Y, Huang J, Sun X, Kang R, Tang D: Ferroptosis: process and function. Cell Death Differ 2016, 23(3):369-379.

43. Seibt TM, Proneth B, Conrad M: Role of GPX4 in ferroptosis and its pharmacological implication. Free Radic Biol Med 2019, 133:144-152.

44. Seiler A, Schneider M, Förster H, Roth S, Wirth EK, Culmsee C, Plesnila N, Kremmer E, Rådmark O, Wurst W et al: Glutathione peroxidase 4 senses and translates oxidative stress into 12/15-lipoxygenase dependent- and AIF-mediated cell death. Cell metabolism 2008, 8(3):237-248.

45. Ingold I, Berndt C, Schmitt S, Doll S, Poschmann G, Buday K, Roveri A, Peng X, Porto Freitas F, Seibt T et al: Selenium Utilization by GPX4 Is Required to Prevent Hydroperoxide-Induced Ferroptosis. Cell 2018, 172(3):409-422.e421.

46. Masaldan S, Bush Al, Devos D, Rolland AS, Moreau C: Striking while the iron is hot: Iron metabolism and ferroptosis in neurodegeneration. Free Radic Biol Med 2019, 133:221-233.

47. Weiland A, Wang Y, Wu W, Lan X, Han X, Li Q, Wang J: Ferroptosis and Its Role in Diverse Brain Diseases. Mol Neurobiol 2019, 56(7):4880-4893. 
48. Bartzokis G, Sultzer D, Mintz J, Holt LE, Marx P, Phelan CK, Marder SR: In vivo evaluation of brain iron in Alzheimer's disease and normal subjects using MRI. Biological psychiatry 1994, 35(7):480-487.

49. Bartzokis $\mathrm{G}$, Tishler TA: MRI evaluation of basal ganglia ferritin iron and neurotoxicity in Alzheimer's and Huntingon's disease. Cellular and molecular biology (Noisy-le-Grand, France) 2000, 46(4):821-833.

50. Bartzokis G, Tishler TA, Shin IS, Lu PH, Cummings JL: Brain ferritin iron as a risk factor for age at onset in neurodegenerative diseases. Annals of the New York Academy of Sciences 2004, 1012:224-236.

51. Pfefferbaum A, Adalsteinsson E, Rohlfing T, Sullivan EV: MRI estimates of brain iron concentration in normal aging: comparison of field-dependent (FDRI) and phase (SWI) methods. Neuroimage 2009, 47(2):493-500.

52. Bilgic B, Pfefferbaum A, Rohlfing T, Sullivan EV, Adalsteinsson E: MRI estimates of brain iron concentration in normal aging using quantitative susceptibility mapping. Neuroimage 2012, 59(3):26252635.

53. Langkammer C, Ropele S, Pirpamer L, Fazekas F, Schmidt R: MRI for iron mapping in Alzheimer's disease. Neuro-degenerative diseases 2014, 13(2-3):189-191.

54. Ghadery C, Pirpamer L, Hofer E, Langkammer C, Petrovic K, Loitfelder M, Schwingenschuh P, Seiler S, Duering $M$, Jouvent $E$ et al: R2* mapping for brain iron: associations with cognition in normal aging. Neurobiol Aging 2015, 36(2):925-932.

55. Tao Y, Wang Y, Rogers JT, Wang F: Perturbed iron distribution in Alzheimer's disease serum, cerebrospinal fluid, and selected brain regions: a systematic review and meta-analysis. $J$ Alzheimers Dis 2014, 42(2):679-690.

56. Caldwell JH, Klevanski M, Saar M, Müller UC: Roles of the amyloid precursor protein family in the peripheral nervous system. Mechanisms of development 2013, 130(6-8):433-446.

57. Huang YA, Zhou B, Wernig M, Sudhof TC: ApoE2, ApoE3, and ApoE4 Differentially Stimulate APP Transcription and Abeta Secretion. Cell 2017, 168(3):427-441 e421.

58. Ward RJ, Zucca FA, Duyn JH, Crichton RR, Zecca L: The role of iron in brain ageing and neurodegenerative disorders. The Lancet Neurology 2014, 13(10):1045-1060.

59. McCarthy RC, Park YH, Kosman DJ: SAPP modulates iron efflux from brain microvascular endothelial cells by stabilizing the ferrous iron exporter ferroportin. EMBO Rep 2014, 15(7):809-815.

60. Wan L, Nie G, Zhang J, Zhao B: Overexpression of human wild-type amyloid- $\beta$ protein precursor decreases the iron content and increases the oxidative stress of neuroblastoma SH-SY5Y cells. $J$ Alzheimers Dis 2012, 30(3):523-530. 
61. Wong BX, Tsatsanis A, Lim LQ, Adlard PA, Bush Al, Duce JA: beta-Amyloid precursor protein does not possess ferroxidase activity but does stabilize the cell surface ferrous iron exporter ferroportin. PLOS One 2014, 9(12):e114174.

62. Morris G, Berk M, Carvalho AF, Maes M, Walker AJ, Puri BK: Why should neuroscientists worry about iron? The emerging role of ferroptosis in the pathophysiology of neuroprogressive diseases. Behav Brain Res 2018, 341:154-175.

63. Chen L, Hambright WS, Na R, Ran Q: Ablation of the Ferroptosis Inhibitor Glutathione Peroxidase 4 in Neurons Results in Rapid Motor Neuron Degeneration and Paralysis. J Biol Chem 2015, 290(47):28097-28106.

64. Dodson M, Castro-Portuguez R, Zhang DD: NRF2 plays a critical role in mitigating lipid peroxidation and ferroptosis. Redox Biol 2019, 23:101107.

65. Abdalkader M, Lampinen R, Kanninen KM, Malm TM, Liddell JR: Targeting Nrf2 to Suppress Ferroptosis and Mitochondrial Dysfunction in Neurodegeneration. Front Neurosci 2018, 12:466.

66. Sun X, Ou Z, Chen R, Niu X, Chen D, Kang R, Tang D: Activation of the p62-Keap1-NRF2 pathway protects against ferroptosis in hepatocellular carcinoma cells. Hepatology 2016, 63(1):173-184.

67. Liu Z, Lv X, Song E, Song Y: Fostered Nrf2 expression antagonizes iron overload and glutathione depletion to promote resistance of neuron-like cells to ferroptosis. Toxicol Appl Pharmacol 2020, 407:115241.

68. Fan Z, Wirth AK, Chen D, Wruck CJ, Rauh M, Buchfelder M, Savaskan N: Nrf2-Keap1 pathway promotes cell proliferation and diminishes ferroptosis. Oncogenesis 2017, 6(8):e371.

69. Zhao Y, Lu J, Mao A, Zhang R, Guan S: Autophagy Inhibition Plays a Protective Role in Ferroptosis Induced by Alcohol via the p62-Keap1-Nrf2 Pathway. J Agric Food Chem 2021, 69(33):96719683.

70. Li S, Zheng L, Zhang J, Liu X, Wu Z: Inhibition of ferroptosis by up-regulating Nrf2 delayed the progression of diabetic nephropathy. Free Radic Biol Med 2021, 162:435-449.

71. Lv $\quad L, Y u J, X u X, L u T, X u F$ : Eriodictyol inhibits high glucose-induced oxidative stress and inflammation in retinal ganglial cells. J Cell Biochem 2019, 120(4):5644-5651.

72. Jing X, Shi H, Zhu X, Wei X, Ren M, Han M, Ren D, Lou H: Eriodictyol Attenuates $\beta$-Amyloid 25-35 Peptide-Induced Oxidative Cell Death in Primary Cultured Neurons by Activation of Nrf2. Neurochemical research 2015, 40(7):1463-1471.

73. Li YC, Pirro AE, Amling M, Delling G, Baron R, Bronson R, Demay MB: Targeted ablation of the vitamin $\mathrm{D}$ receptor: an animal model of vitamin D-dependent rickets type II with alopecia. Proceedings of 
the National Academy of Sciences of the United States of America 1997, 94(18):9831-9835.

74. Chen L, Yang R, Qiao W, Zhang W, Chen J, Mao L, Goltzman D, Miao D: 1,25-Dihydroxyvitamin D exerts an antiaging role by activation of Nrf2-antioxidant signaling and inactivation of p16/p53senescence signaling. Aging Cell 2019, 18(3):e12951.

\section{Figures}


A<smiles>O=C1CC(c2ccc(O)c(O)c2)Oc2cc(O)cc(O)c21</smiles>

D

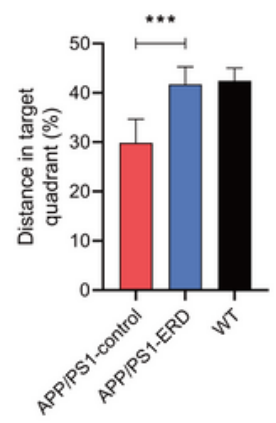

B

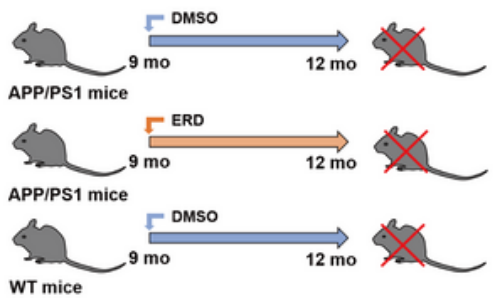

E

F

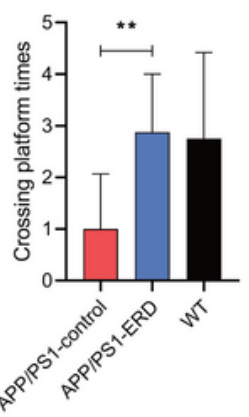

C

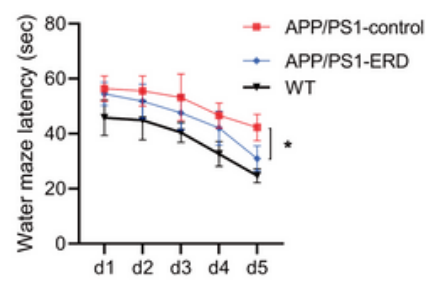

H

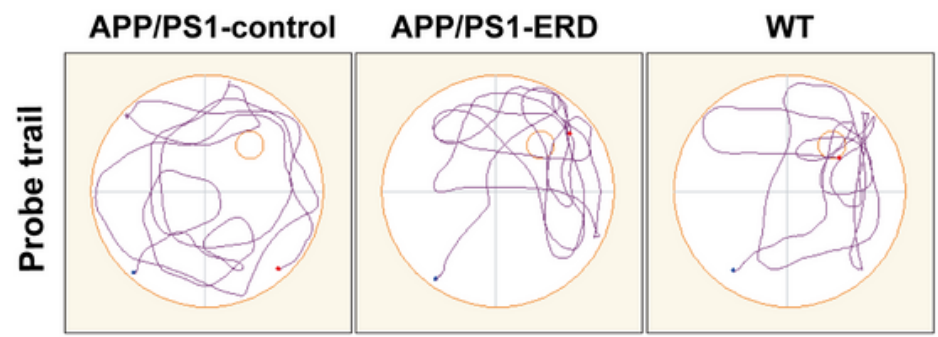

K

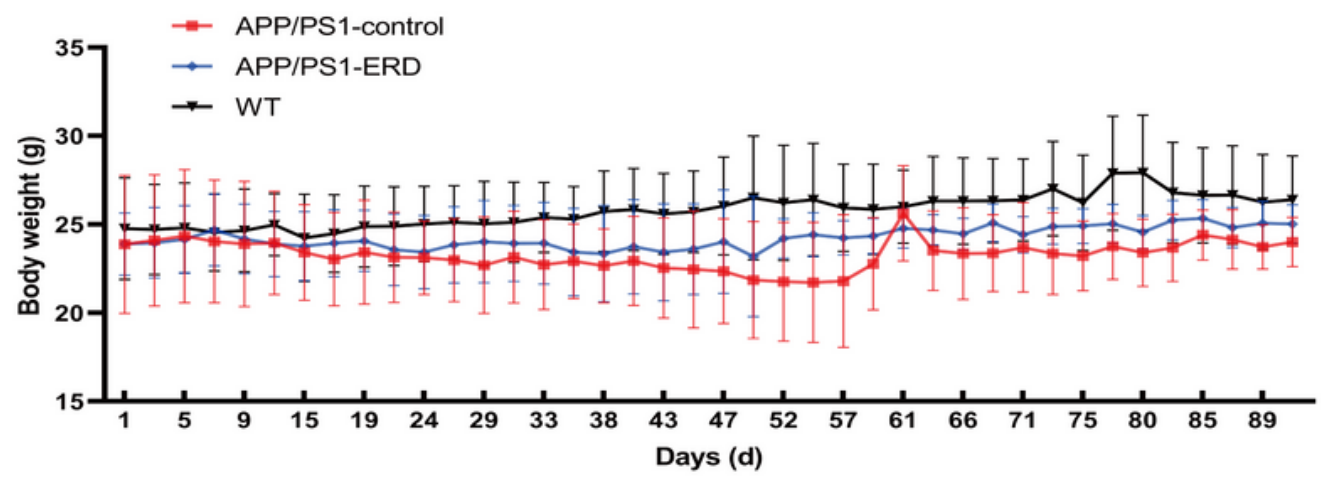

Figure 1

Eriodictyol ameliorates cognitive deficits in APP/PS1 mice tested by Morris water maze. (A) The chemical structure of eriodictyol. (B) Animal grouping and experimental scheme for the effect of eriodictyol on memory damage in APP/PS1 mice. (C) Escape lantency of mice during the fifth day of platform trials. (D) The percentage of distance in target quadrant. (E) The time spent in target quadrant and (F) the number of platform crossings in the probe trials were determined. (G) The swimming speed of mice in Morris 
water maze. $(\mathrm{H})$ The track of the mouse in Morris water maze also recorded. The dose of eriodictyol is 50 $\mathrm{mg} / \mathrm{kg}$, and intraperitoneal injection three times a week for 3 months three times a week for 3 months. Data are presented as means $\pm S D, n=6 .{ }^{*} P<0.05$, ${ }^{* *} P<0.01$, ${ }^{* *} P<0.001$, vs. APP/PS1-control group.

A

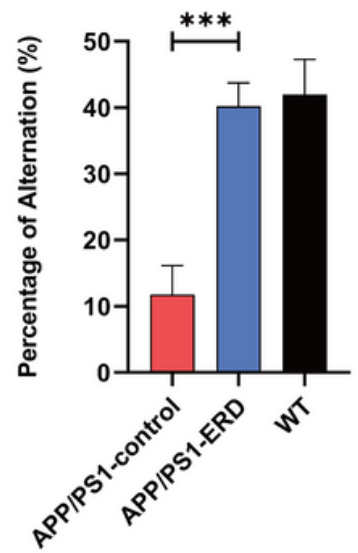

$\mathbf{E}$

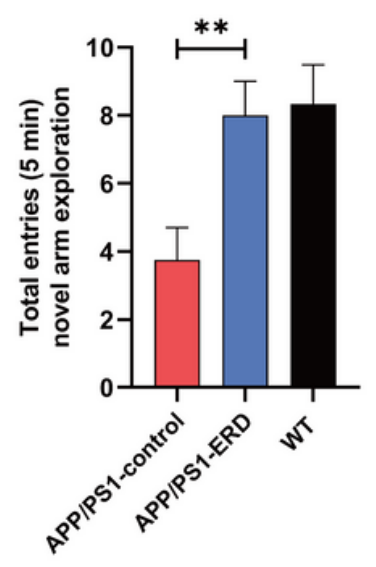

B

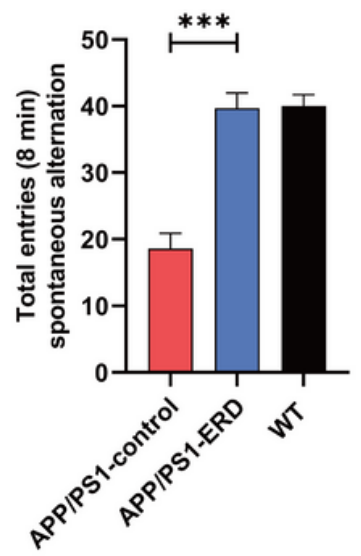

$\mathbf{F}$

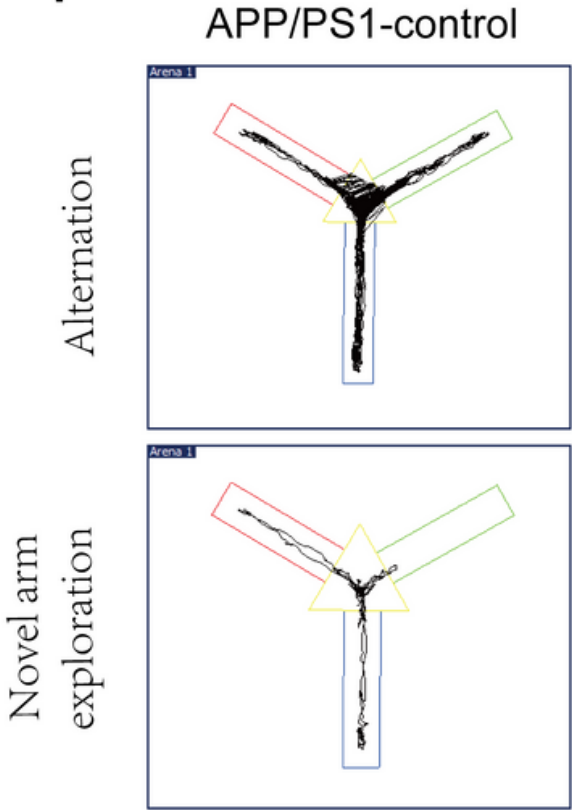

C

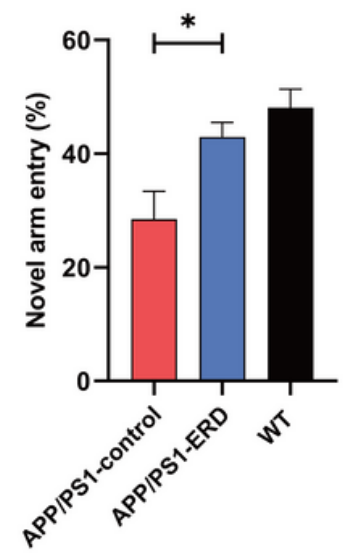

D

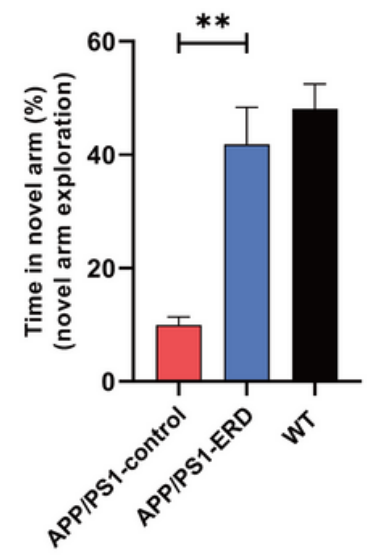

\section{Figure 2}

Eriodictyol ameliorates cognitive deficits in APP/PS1 mice determined by Y-maze tests. (A) Percentage of alternation and (B) total entries during $8 \mathrm{~min}$ was recorded in spontaneous alternation tests. and in novel arm exploration tests. (C) The percentage of novel entries. (D) The time spent in novel arm and (E) the total entries during the five minutes were recorded. $(F)$ The track of mouse in Y-maze during spontaneous alternation tests and novel arm exploration tests. Data are presented as means $\pm S E M, n=6$. ${ }^{P}<0.05$, ** $P<0.01, * * * P<0.001$, vs. APP/PS1-control group.

Figure 3 
Eriodictyol alleviates $A \beta$ aggregation and Tau hyper-phosphorylation in APP/PS1 mice. (A) A aggregation was measured by Congo red staining. (B) The level of $A \beta$ and $p$-Tau in the brain of mouse was tested by IHE assay. (C-D) The expression of $p$-Tau, Tau and $A \beta$ in cortex and hippocampus of mice were tested by Western blot analysis $\beta$-actin was used as loading control. The dose of eriodictyol is 50 $\mathrm{mg} / \mathrm{kg}$, and intraperitoneal injection three times a week for 3 months.

A

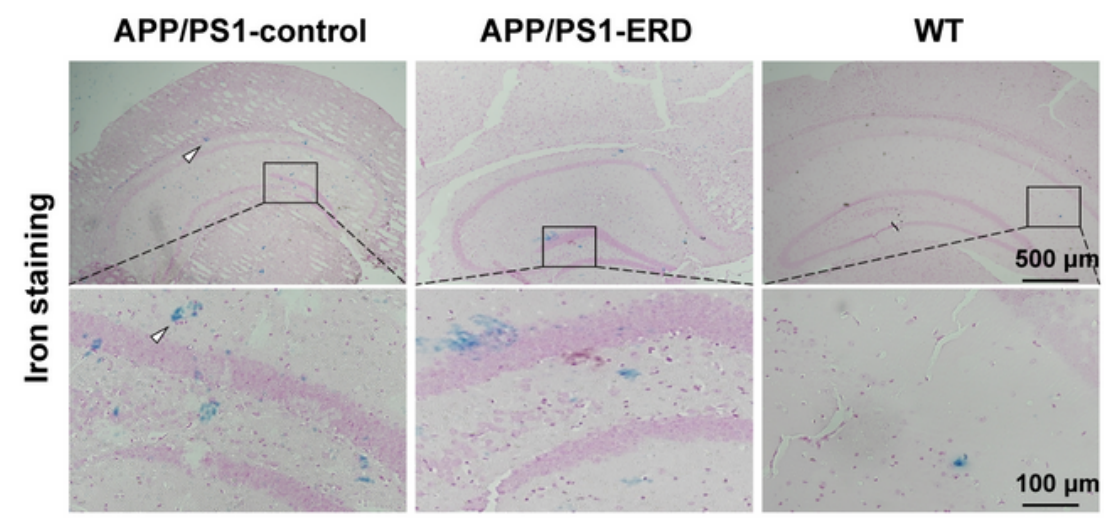

C
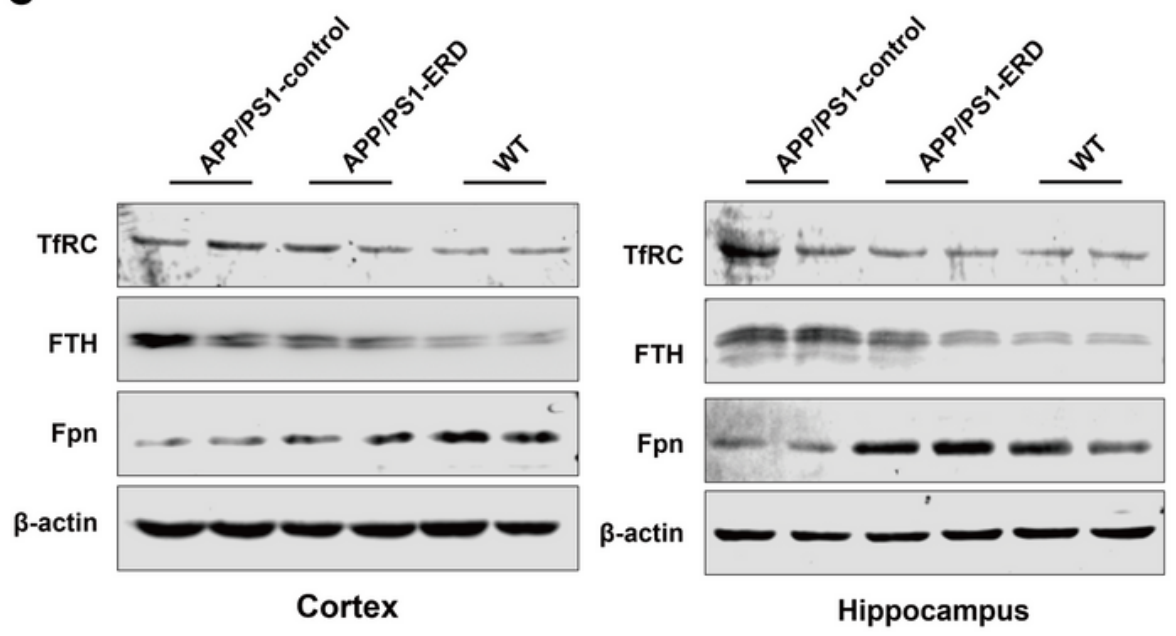

E

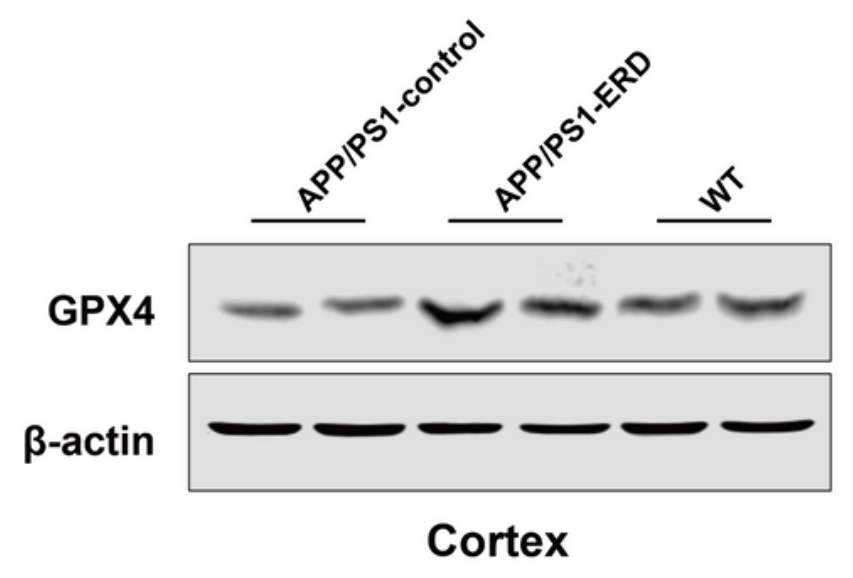

B

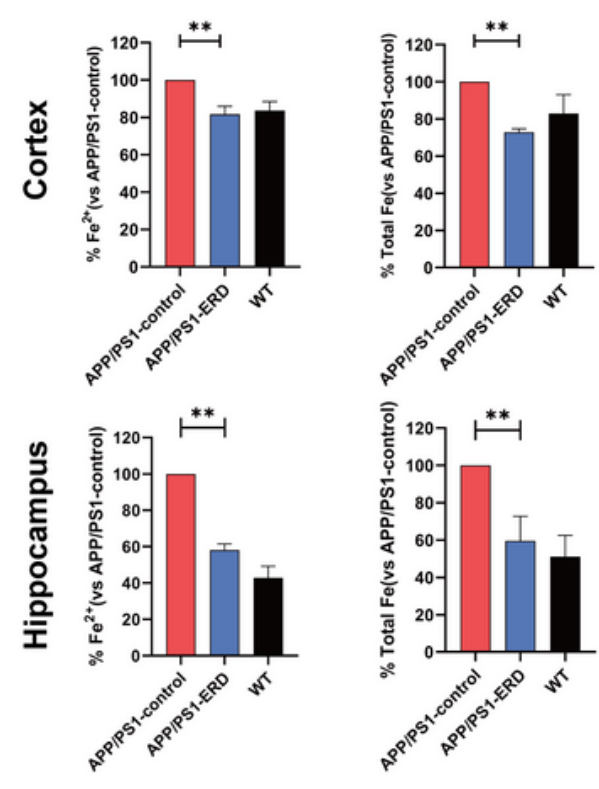

D
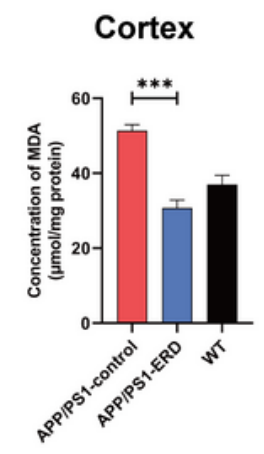

Hippocampus

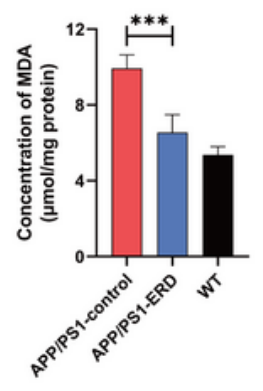

Figure 4 
Eriodictyol inhibits ferroptosis in brain of APP/PS1 mice. (A) Accumulation of iron in brain of mice was strained by Prussian blue staining. (B) The level of ferrous iron and total iron in cortex and hippocampus of mice were dectected using Iron Assay Kit, respectively. (C) The expression of TfRC, FTH and Fpn in cortex and hippocampus of mice brain was measured by Western blot and $\beta$-actin was used as loading control. (D) MDA content in cortex and hippocampus of mice brain was detected using Lipid Peroxidation MDA Assay Kit. (E) The expression level of GPX4 in cortex and hippocampus of mice brain was measured by Western blot. The data are shown as the mean $\pm \mathrm{SD}$ of three experiments. ${ }^{*} \mathrm{P}<0.05,{ }^{*} \mathrm{P}<$ 0.01 , *** $\mathrm{P}<0.001$ compared with the control group.

A

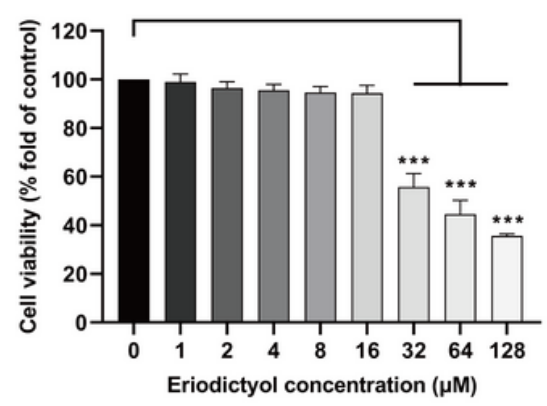

B

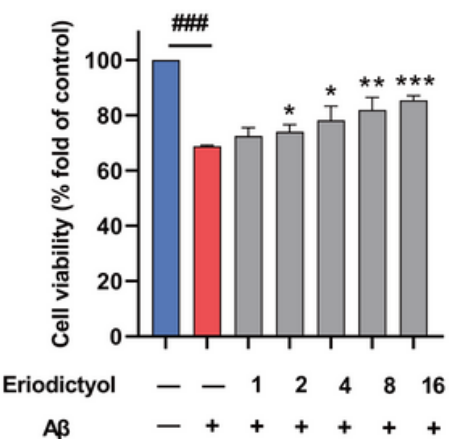

C

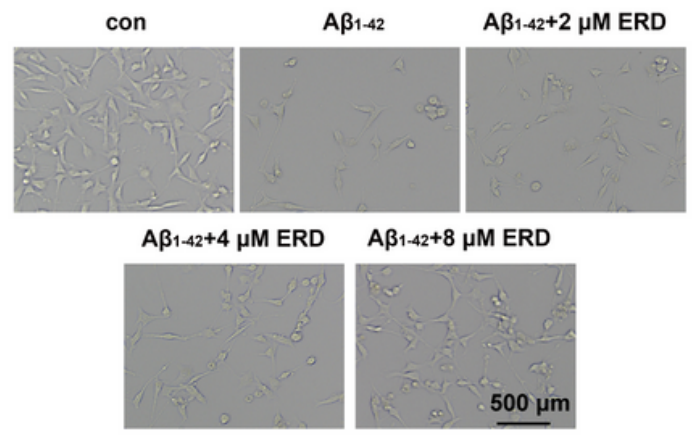

D
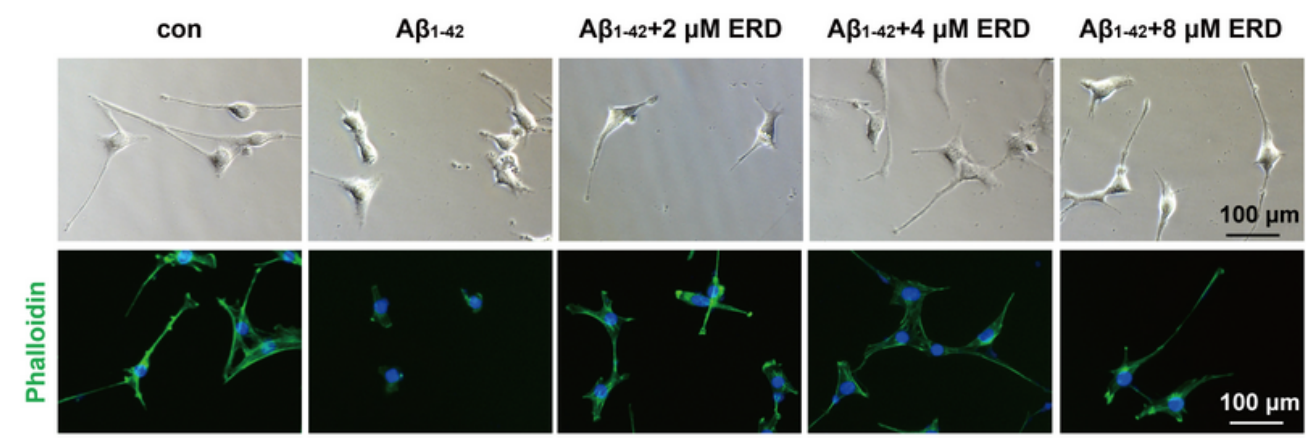

E

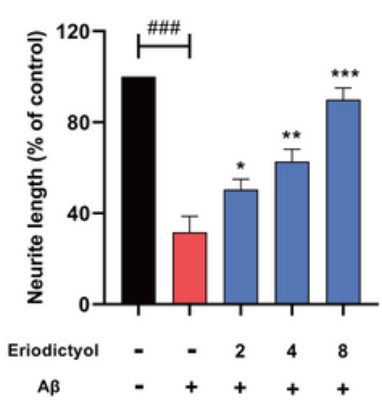

G
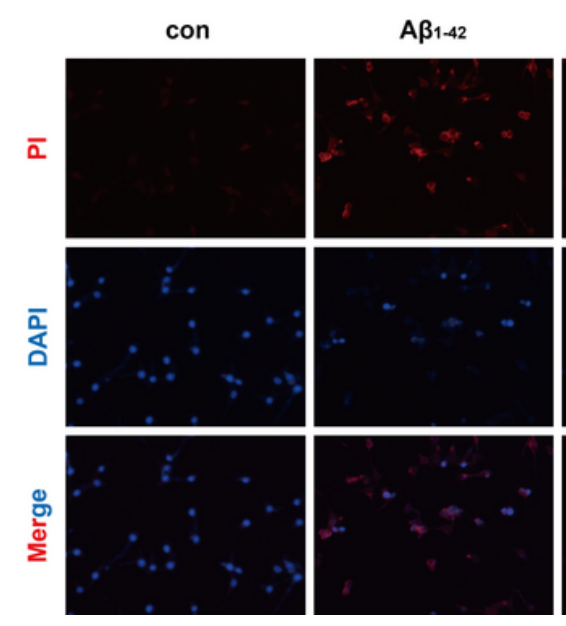
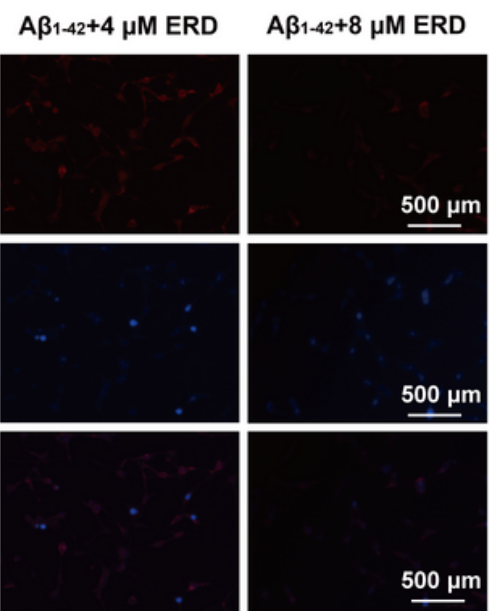

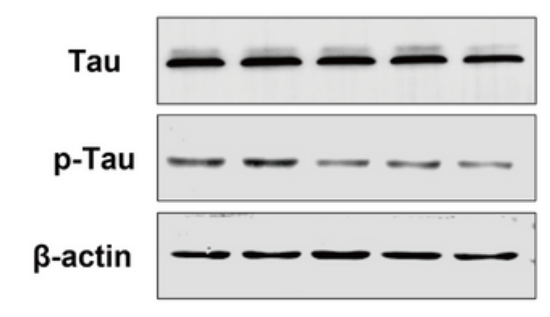

$\begin{array}{llllll}\text { Eriodictyol } & - & - & 2 & 4 & 8\end{array}$

A $\beta$ 


\section{Figure 5}

Eriodictyol attenuated the cytotoxicity and Tau hyper-phosphorylation induced by AB1-42 oligomer in HT22 cells. (A) Viability of HT-22 cells was measured by CCK-8 assay to explore the cytotoxicity of eriodictyol on HT-22 cells. Cells were treated with eriodictyol $(0,2,4,8,16,32,64$ or $128 \mu \mathrm{M})$ for $48 \mathrm{~h}$. (B) Viability of HT-22 cells was measured by CCK-8 assay. HT-22 cells were pre-exposed to eriodictyol $(0,1,2$, $4,8$ or $16 \mu \mathrm{M})$ for $2 \mathrm{~h}$, and then treated with $A \beta 1-42$ oligomer $(20 \mu \mathrm{M})$ for $48 \mathrm{~h}$. (C) The state and number of HT-22 cells were observed under microscope. HT-22 cells were pre-treated with eriodictyol $(0,2,4$ or 8 $\mu \mathrm{M})$ and $20 \mu \mathrm{M}$ AB1-42 oligomer for 48h. (D) The cytoskeleton of HT-22 cells was stained with FITCphalloidin. HT-22 cells were treated with eriodictyol $(0,2,4$ or $8 \mu \mathrm{M})$ and $20 \mu \mathrm{M}$ Aß1-42 oligomer for $48 \mathrm{~h}$ after induced by $10 \mu \mathrm{M}$ RA for 3 days. (E) Quantification of the neurite length of HT-22 cells by ImageJ software. (F) Cell death detected by Propidium lodide assay. (G) The expression of Tau and p-Tau was measured by Western blot and $\beta$-actin was used as loading control. The data are shown as the mean \pm SD of three experiments. ${ }^{*} P<0.05,{ }^{* *} P<0.01$, ${ }^{\star \star \star} P<0.001$ compared with the $A \beta 1-42$ oligomer group. \# $\mathrm{P}<0.05$, \#\# $\mathrm{P}<0.01$, \#\#\# $\mathrm{P}<0.001$ compared with the control group. 
A

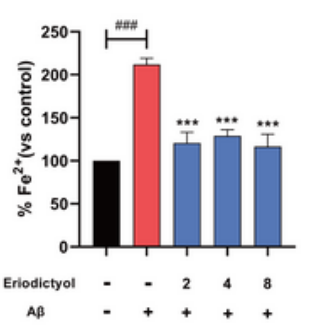

C

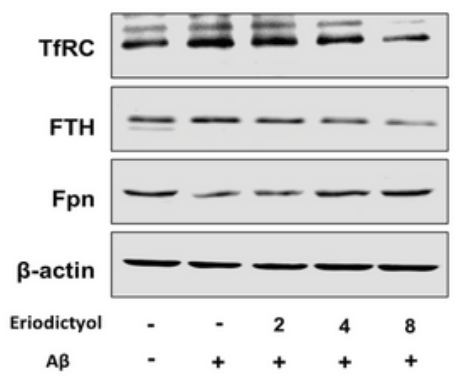

E
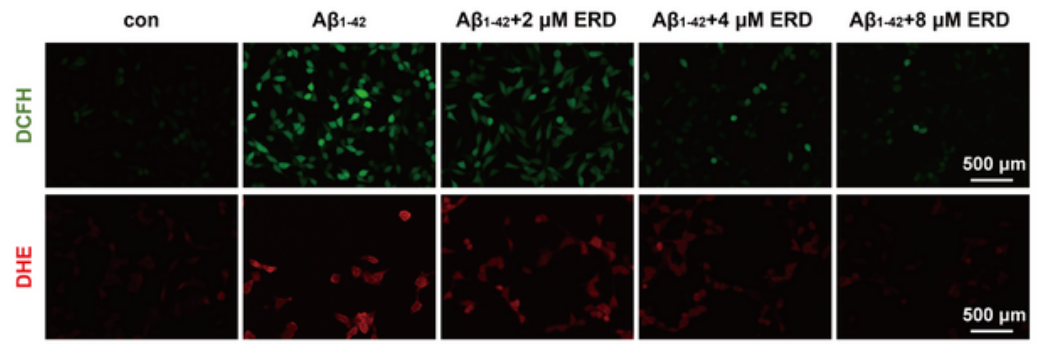

F

D

$500 \mu \mathrm{m}$
G

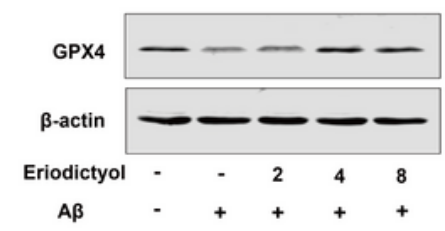

H

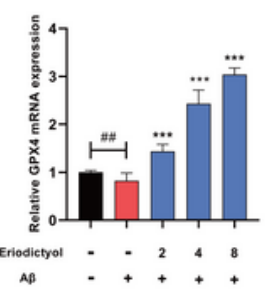

B
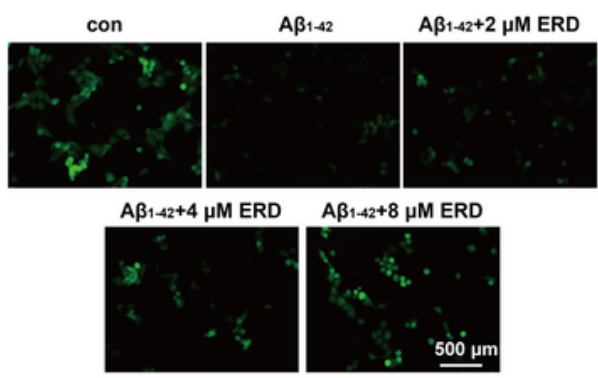

Calcein-AM
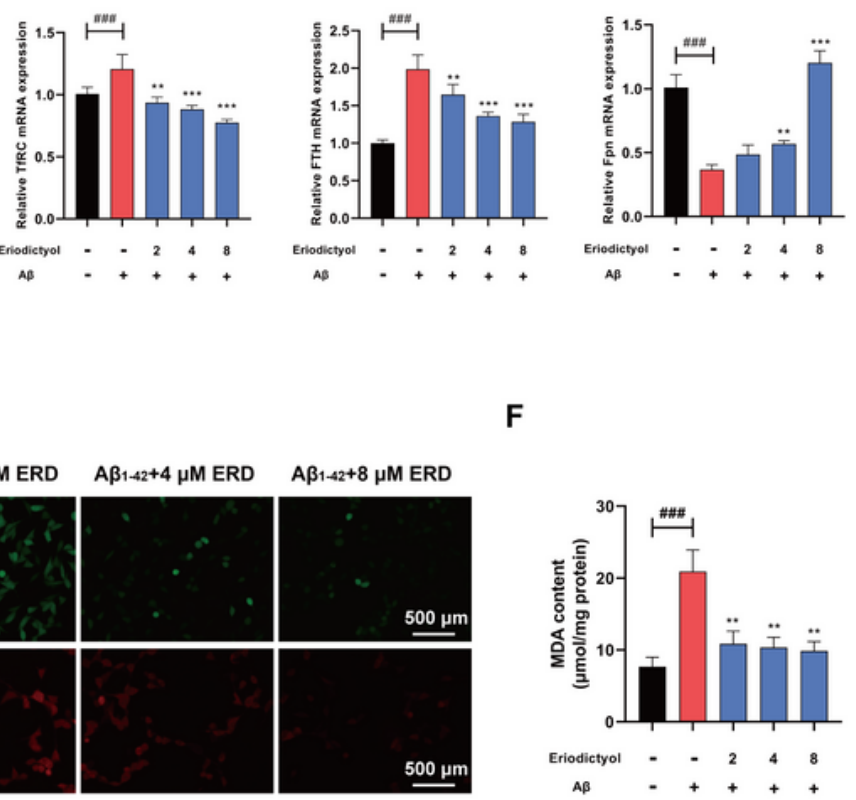

I

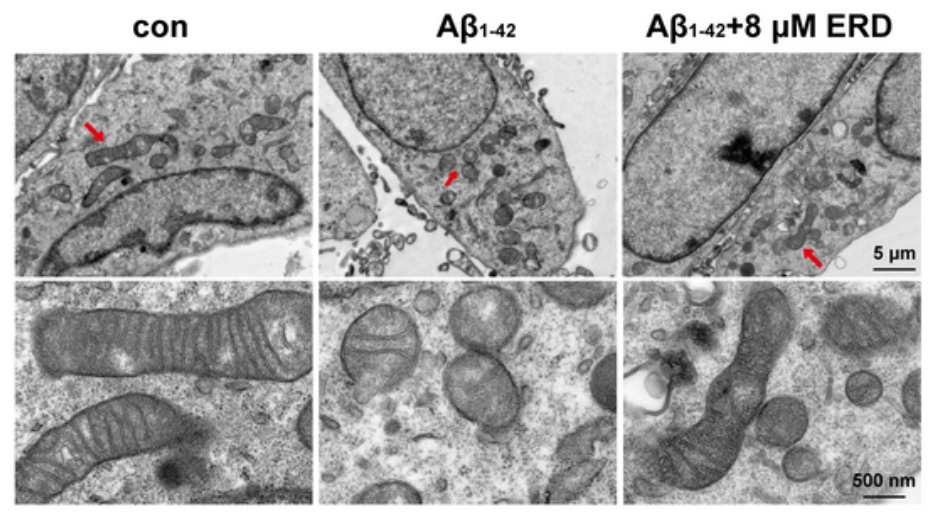

Figure 6

Eriodictyol inhibits ferroptosis induced by A 1 1-42 oligomer in HT-22 cells. (A) The level of ferrous iron and total iron in HT-22 cells were detected using Iron Assay Kit respectively. (B) The content of iron in HT-22 cells was tested by Immunofluorometric Assay with Calcein AM. (C) The expression of TfRC, FTH, and Fpn were measured by Western blot. (D) Those proteins relative mRNA expression level was tested by qPCR. (E) The level of ROS in HT-22 cells was measured using two types of fluorescent probes (DHE and 
DCFH-DA) and (F) the MDA content of HT-22 cells were measured using Lipid Peroxidation MDA Assay Kit. (G) The expression level of GPX4 in HT-22 cells were detected by western blot and $(H)$ its relative mRNA expression level were measured by q-PCR. (I) The morphological features of mitochondria in HT-22 cells were observed under electron microscope. The data are shown as the mean $\pm S D$ of three experiments. ${ }^{*}<<0.05$, $* * P<0.01$, $* \star * P<0.001$ compared with the $A \beta 1-42$ oligomer group. \# $P<0.05$, \#\# $\mathrm{P}<0.01$, \#\#\# $\mathrm{P}<0.001$ compared with the control group.

A
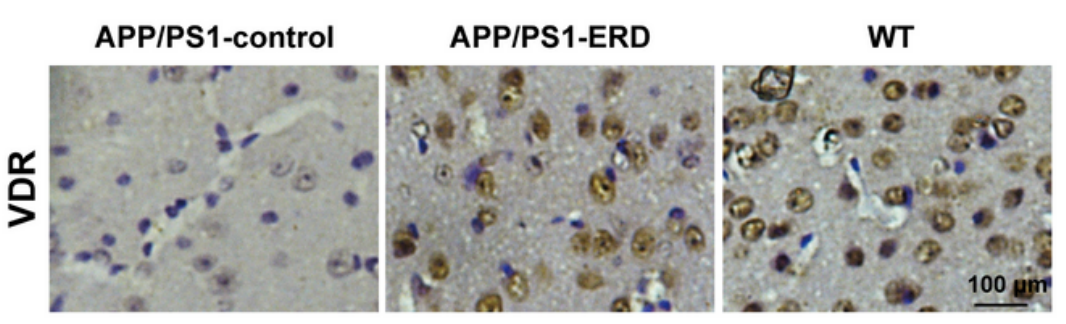

B

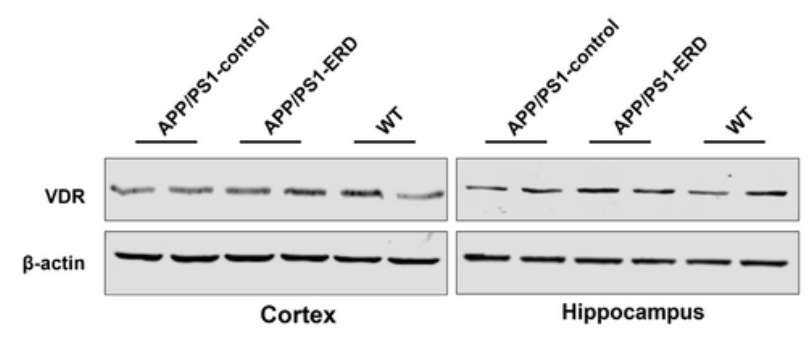

$E$
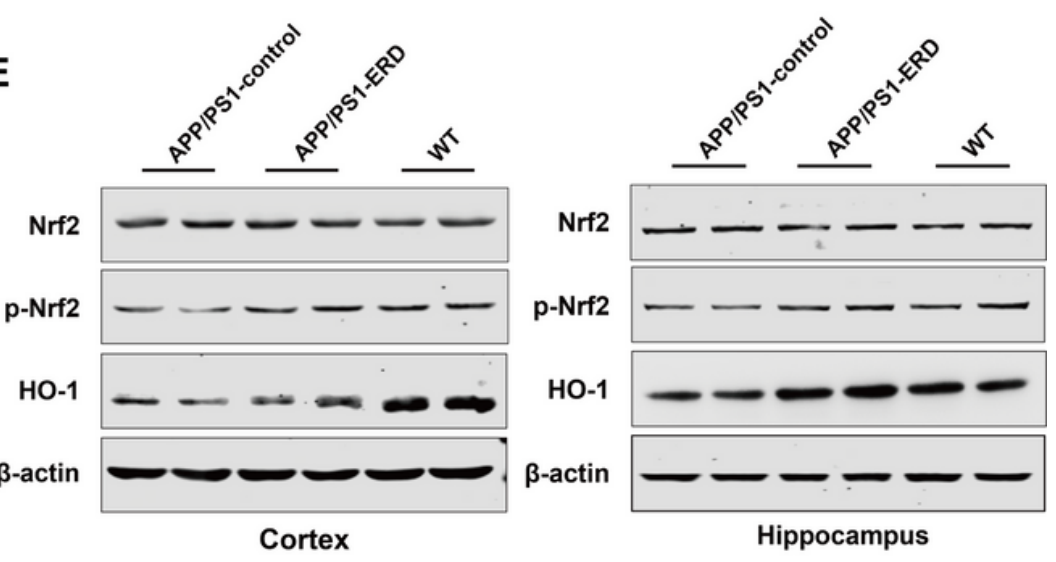

G

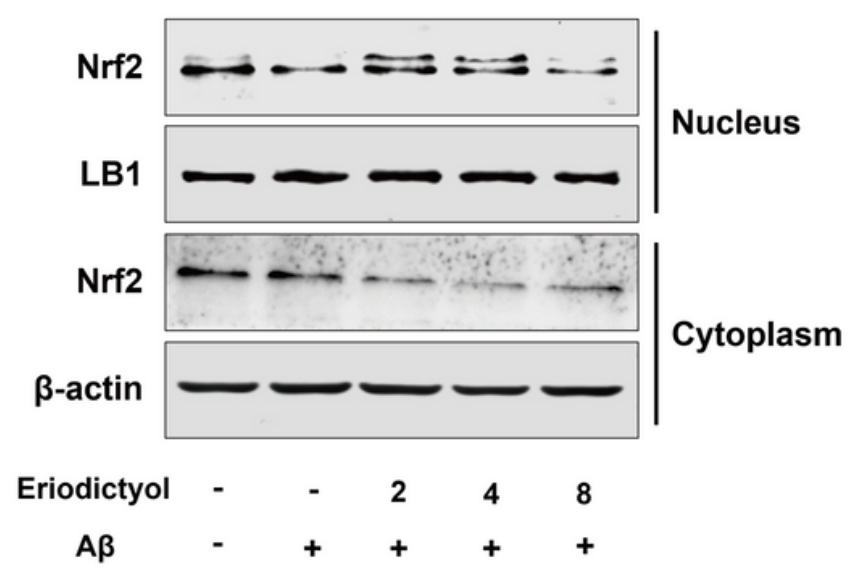

Figure 7

Eriodictyol up-regulates the expression of VDR and activates Nrf2/HO-1 signaling pathway. (A) The expression level of VDR in brain of mice was detected by immunohistochemical analysis. Then (B) the protein expression level of VDR in cortex and hippocampus of mice brain was measured by Western blot. 
(C) The expression level of VDR in HT-22 cells was measured by western blot and (D) its relative mRNA expression level was detected by q-PCR. (E) The expression level of Nrf2, p-Nrf2 and HO-1 in cortex and hippocampus of mice brain were measured by Western blot. (F) The expression level of Nrf2, p-Nrf2 and HO-1 in HT-22 cells were measured by western blot. (G) The expression level of Nrf2 in nucleus and cytoplasm of HT-22 cells were detected by western blot and Lamin B1 or $\beta$-actin were used as loading control, respectively. The data are shown as the mean \pm SD of three experiments. ${ }^{*} P<0.05$, $* \star P<0.01$, $\star \star \star P<0.001$ compared with the A 1 1-42 oligomer group. \# $\mathrm{P}<0.05$, \#\# $\mathrm{P}<0.01$, \#\#\# $\mathrm{P}<0.001$ compared with the control group. 
A

B

$\beta$-actin
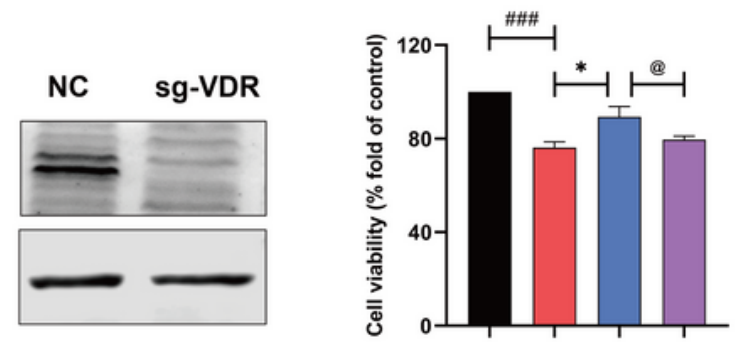

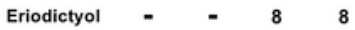

$A \beta-+++$

sg-VDR - - - +

D

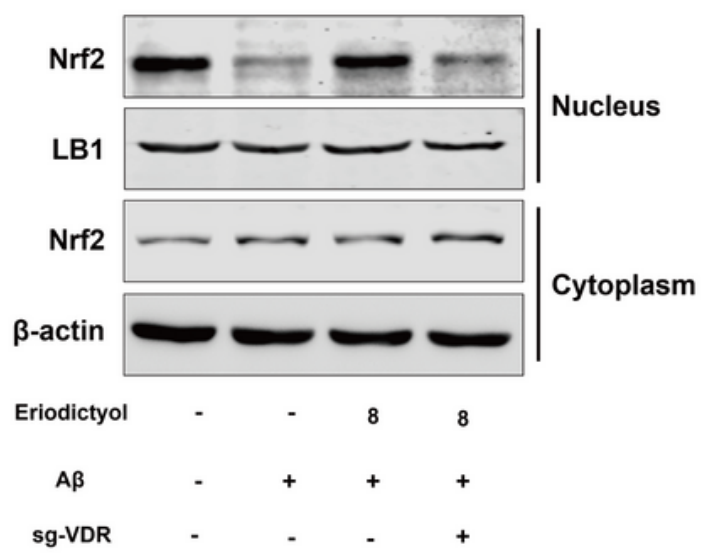

$\mathbf{F}$
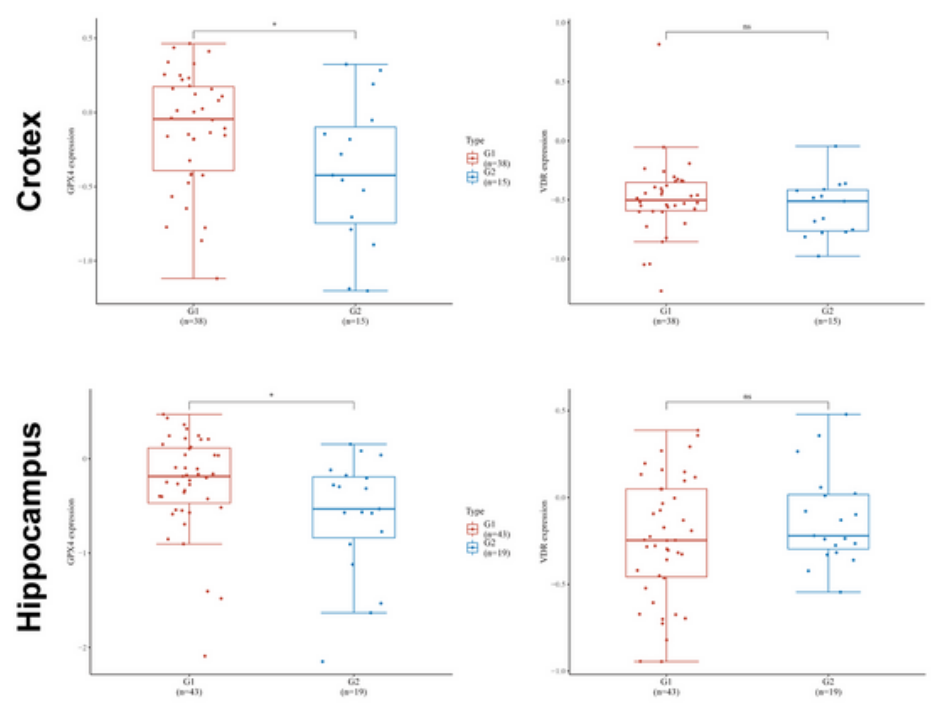

C

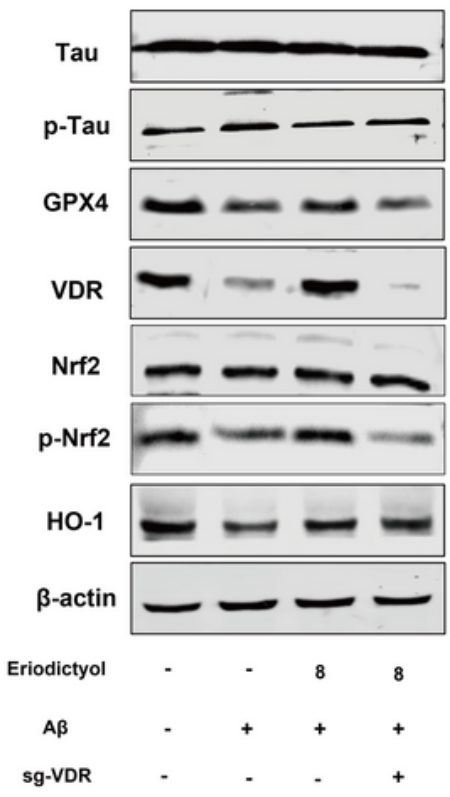

E

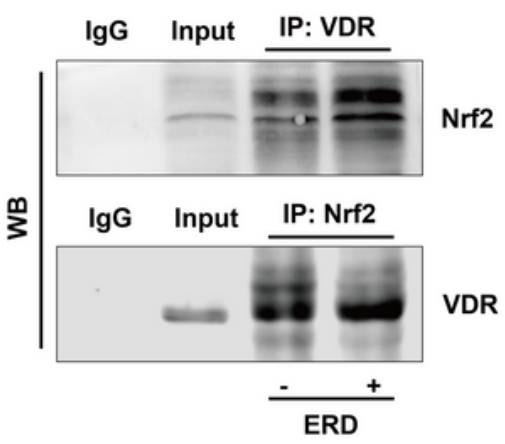

G

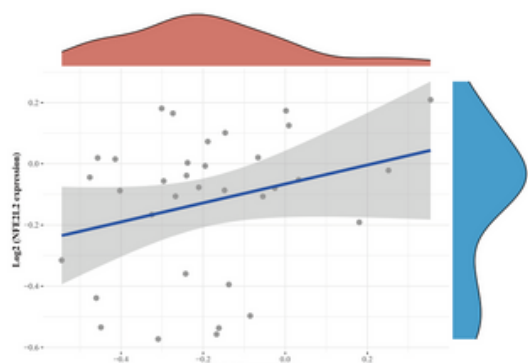

\section{Figure 8}

VDR is the key mediator in the regulation of Nrf2/HO-1 signaling pathway by eriodictyol. (A) VDR was knockout successfully by CRISPR/CAS9. (B) Viability of HT-22 cells was tested by CCK-8 assay. AB1-42 oligomer $(20 \mu \mathrm{M})$-induced normal or knockout VDR in HT-22 cells were treated with or without eriodictyol $(8 \mu \mathrm{M})$ for $48 \mathrm{~h}$. (C) The expression level of Tau, p-Tau, GPX4, VDR, Nrf2, p-Nrf2 and HO-1 in HT-22 cells and sgVDR HT-22 cells were measured by Western blot. (D) The expression level of Nrf2 in nucleus and 
cytoplasm of HT-22 cells and sgVDR HT-22 cells were measured by western blot. (E) The interaction between VDR and Nrf2 was explored by coimmunoprecipitation assay. (F) The VDR and GPX4 expression levels in cortex and hippocampus between normal elderly (G1) and AD patients (G2) were explored by gene difference analysis. $(G)$ The relationship of Nrf2 and VDR in humans, $R=0.22, P=0.208$. The data are shown as the mean $\pm S D$ of three experiments. ${ }^{*} \mathrm{P}<0.05$ compared with the control group. \#\#\# $\mathrm{P}<$ 0.001 compared with A 1-42 oligomer group. @ P $<0.05$ compared with knockout VDR group.

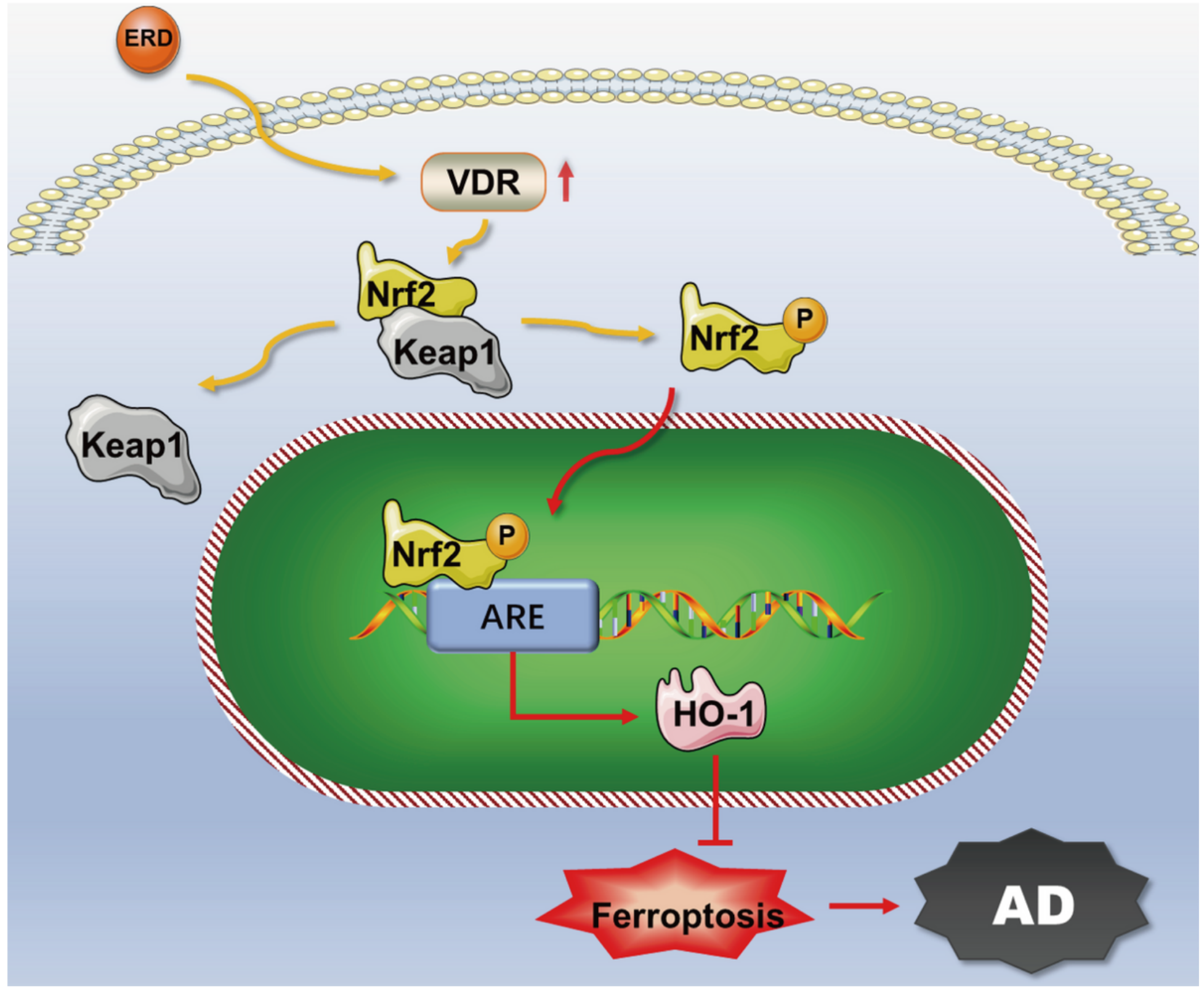

\section{Figure 9}

The mechanism of eriodictyol inhibit AD. Eriodictyol alleviated cognitive injury by preventing neuron cell from ferroptosis, its mechanism may be connected with the activation of Nrf2/HO-1 mediated by VDR. 